\title{
Rosetta lander Philae: Flight Dynamics analyses for landing site selection and post-landing operations
}

\author{
Eric Jurado ${ }^{\mathrm{a}, *}$, Thierry Martin ${ }^{\mathrm{a}}$, Elisabet Canalias ${ }^{\mathrm{a}}$, Alejandro Blazquez ${ }^{\mathrm{a}}$, Romain Garmier ${ }^{\mathrm{b}}$, \\ Thierry Ceolin ${ }^{\mathrm{b}}$, Philippe Gaudon ${ }^{\mathrm{a}}$, Cedric Delmas ${ }^{\mathrm{a}}$, Jens Biele ${ }^{\mathrm{c}}$, Stephan Ulamec ${ }^{\mathrm{c}}$, \\ Emile Remetean $^{\mathrm{a}}$, Alex Torres ${ }^{\mathrm{a}}$, Julien Laurent-Varin ${ }^{\mathrm{a}}$, Benoit Dolives ${ }^{\mathrm{d}}$, Alain Herique ${ }^{\mathrm{e}}$, \\ Yves Rogez ${ }^{\mathrm{e}}$, Wlodek Kofman ${ }^{\mathrm{e}}$, Laurent Jorda ${ }^{\mathrm{f}}$, Vladimir Zakharov ${ }^{\mathrm{g}, \mathrm{j}}$, Jean-François Crifo ${ }^{\mathrm{g}}$, \\ Alexander Rodionov ${ }^{\mathrm{k}}$, P. Heinish ${ }^{\mathrm{h}}$, Jean-Baptiste Vincent ${ }^{\mathrm{i}}$ \\ ${ }^{a}$ CNES, 18 Avenue E. Belin, Toulouse 31401, France \\ ${ }^{\mathrm{b}}$ CS, 5 rue brindejonc des moulinais B 15872, Toulouse 31506, France \\ ${ }^{c}$ DLR, Linder Höhe, Köln 51147, Germany \\ d Magellium, 24 rue Hermes, Ramonville Saint Agne 31520, France \\ e University Grenoble Alpes, IPAG, Grenoble F-38000, France \\ ${ }^{\mathrm{f}}$ LAM, 38, rue Joliot-Curie, Marseille 13388, France \\ ${ }^{\mathrm{g}}$ LATMOS, 11 Boulevard d'Alembert, Guyancourt 78280, France \\ h IGEP, Technical University Mendelssohnstr., Braunschweig 38106, Germany \\ ${ }^{i}$ Max Planck Institut für Sonnensystemforschung, Göttingen, Germany \\ ${ }^{\mathrm{j}}$ LESIA, France \\ ${ }^{\mathrm{k}}$ FSUE RFNC-VNIIEF, Russian Federation
}

\section{A R T I C L E I N F O}

\section{Article history:}

Received 9 October 2015

Received in revised form

29 March 2016

Accepted 29 March 2016

Available online 13 April 2016

\section{Keywords:}

Rosetta

Philae

Flight Dynamics

Landing

Trajectory

Attitude

\begin{abstract}
A B S T R A C T
On the 12th of November 2014, The Rosetta Lander Philae became the first spacecraft to softly land on a comet nucleus. Due to the double failure of the cold gas hold-down thruster and the anchoring harpoons that should have fixed Philae to the surface, it spent approximately two hours bouncing over the comet surface to finally come at rest one km away from its target site. Nevertheless it was operated during the $57 \mathrm{~h}$ of its First Science Sequence. The FSS, performed with the two batteries, should have been followed by the Long Term Science Sequence but Philae was in a place not well illuminated and fell into hibernation. Yet, thanks to reducing distance to the Sun and to seasonal effect, it woke up at end of April and on 13th of June it contacted Rosetta again. To achieve this successful landing, an intense preparation work had been carried out mainly between August and November 2014 to select the targeted landing site and define the final landing trajectory. After the landing, the data collected during on-comet operations have been used to assess the final position and orientation of Philae, and to prepare the wake-up. This paper addresses the Flight Dynamics studies done in the scope of this landing preparation from Lander side, in close cooperation with the team at ESA, responsible for Rosetta, as well as for the reconstruction of the bouncing trajectory and orientation of the Lander after touchdown.
\end{abstract}

c 2016 IAA. Published by Elsevier Ltd. All rights reserved.

\section{Introduction}

Rosetta is a cornerstone mission of the European Space Agency (ESA) Horizon 2000 program. It reached its target comet 67P/ Churyumov-Gerasimenko in August 2014 after a 10 years cruise in the Solar System. Its scientific payload consists of 11 instruments including the Philae lander. Rosetta is currently observing its icy nucleus and coma, and when the mission will come to an end in

\footnotetext{
* Corresponding author.

E-mail address: eric.jurado@cnes.fr (E. Jurado).
}

September 2016, the comet will have passed the perihelion and will continue on its way in the Solar System. One of the objectives of the mission was to deliver the Philae lander to the surface of the comet nucleus. This objective was reached on 12th of November 2014, when the spacecraft achieved the first-ever soft landing on a comet nucleus. Due to a double failure of the hold-down thrust and of the anchoring harpoons, Philae bounced off the comet surface and came to rest only after $1 \mathrm{~h} 50 \mathrm{~min}$. Yet it was able to carry out its main mission and most of its 10 instruments made the first in-situ analyses of a comet.

Rosetta is operated by ESA from Rosetta Mission Operation Center (RMOC) at ESOC, Darmstadt. Rosetta science operation 
Table 1

Timeline of Philae LSSP.

\begin{tabular}{|c|c|c|c|}
\hline Days to Landing & $\begin{array}{l}\text { Date/min distance to } \\
\text { comet }\end{array}$ & RLGS productions & RMOC analysis \\
\hline L-79 & $24 / 08 / 201450 \mathrm{~km}$ & RLGS proposed 5 candidate landing sites to RMOC & $\begin{array}{l}\text { RMOC checked feasibility of the proposed } \\
\text { landing sites. }\end{array}$ \\
\hline L-58 & $14 / 09 / 201430 \mathrm{~km}$ & RLGS proposed the nominal and backup landing sites to RMOC. & RMOC computed the operational trajectory \\
\hline L-30 & $12 / 10 / 201410 \mathrm{~km}$ & $\begin{array}{l}\text { RLGS confirmed the choice of the nominal landing site. Beginning of } \\
\text { operational preparation }\end{array}$ & RMOC updated the operational trajectory \\
\hline
\end{tabular}

planning is performed at Rosetta Science Ground Segment (RSGS) at ESAC, Villafranca near Madrid. Philae is operated from the LCC (Lander Control Center) at DLR, Cologne, and the SONC (Science Operations and Navigation Center) at CNES, Toulouse. Both centers constitute the Rosetta Lander Ground Segment (RLGS). The responsibility for Lander delivery lies at ESA, nevertheless, RLGS is in charge of proposing to ESA the Philae Landing Site, and there have been close cooperation between all the partners to achieve the challenging task of landing on a comet.

\section{The landing site selection process: overview}

The selection of the Landing Site was a complex and critical process that was conducted from July to October 2014 (cf. Table 1). The scientific interest of the Landing Site has of course been a driver for the selection, yet some technical constraints due to Lander and Orbiter design and characteristics had to be taken into account as well. Obviously, safe landing had the highest priority. For further description see for example [5] or [1].

To avoid potentially strong outgassing of the comet, the landing date should absolutely occur before the comet reached the heliocentric distance of $3 \mathrm{AU}$ and the landing date was fixed at 12th November 2014

Rosetta entered into orbit around the comet in August 2014 and before this date very few was known about Comet ChuryumovGerasimenko. It means that the landing site selection process (LSSP) had to be achieved at the same time as Rosetta was collecting data. So the LSSP was built to take into account the availability and accuracy of comet models necessary to compute landing trajectories (cf. Table 2).

Several teams were responsible for producing models with some redundancy and SONC decided to use all available models. This choice allows performing cross validation between models and sensitivity analysis of the results with respect to the input data. The drawback is that it induces more combination cases to be studied and possible conflicts that require careful analysis. Nevertheless, the final operational trajectories were computed using the RMOC (ESOC) models.

\section{Philae separation, descent and landing scenario}

\subsection{Rosetta constraints}

The Rosetta constraints affecting the delivery and descent trajectory are mainly related to the safety and the navigability of the orbiter for the delivery scenario. For instance, the distance from the orbiter to the comet was never allowed to be smaller than $5 \mathrm{~km}$. The relative navigation of the Orbiter around the comet is performed thanks to optical navigation based on the NAVCAM measurements. In order to ensure proper illumination of the comet the sun beta angle should stay between $0^{\circ}$ and $20^{\circ}$ whereas the phase angle shall be maintained between $0^{\circ}$ and $135^{\circ}$. Furthermore, terminator orbits minimize the effect of the outgassing
Table 2

Comet models purpose and providers for landing site selection.

\begin{tabular}{lll}
\hline Model & Source & Purpose \\
\hline Comet Ephemerides & RMOC & $\begin{array}{l}\text { Illumination analysis } \\
\text { Trajectory analysis }\end{array}$ \\
Rotational parameters & RMOC & $\begin{array}{l}\text { Define the transformation EME } \\
\text { 2000 to Comet fixed frame }\end{array}$ \\
Global DTM & OSIRIS & Illumination analysis \\
& RMOC & Trajectory analysis \\
Local DTM & OSIRIS & Local slope analysis \\
Gravity Field & OSIRIS & Trajectory analysis \\
Outgassing & CNES GRGS & Trajectory analysis \\
Boulder distribution & RMOC & Risk assessment \\
Rosetta and Philae & LATMOS & Trajectory analysis and cross \\
trajectories & RMOC & check \\
\hline
\end{tabular}

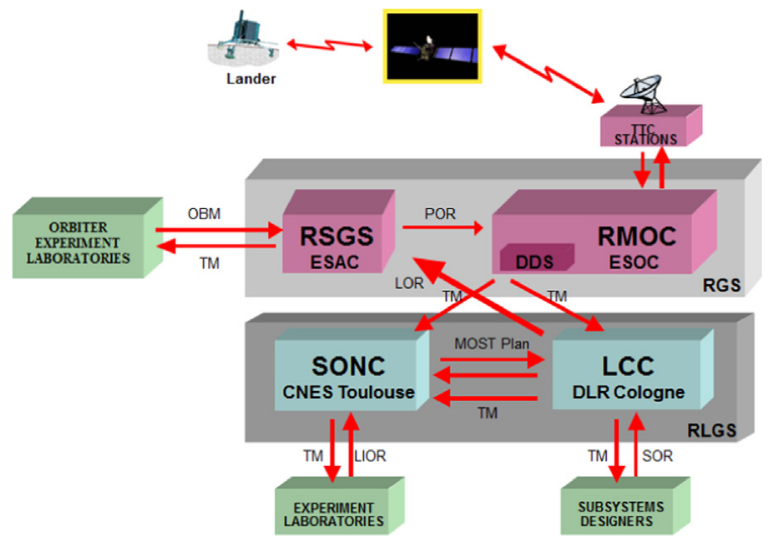

Fig. 1. Lander and orbiter science and control centers.

on the spacecraft, so these were the types of orbits flown at minimal distances.

\subsection{Philae descent and landing equipment}

The Philae Lander has a power system including a solar generator, a central data management system and an S-band communications system, using the Rosetta orbiter as relay. Philae carries a flywheel to stabilize the lander around its $Z$ axis during descent, and a thruster (Active Descent System) with a total $\Delta V$ capability of $1.85 \mathrm{~m} / \mathrm{s}$. It was planned to activate the ADS to perform a hold-down thrust after touchdown. The Philae Landing Gear consists of a foldable tripod with legs and feet and a central structure hosting several mechanisms to execute the various LG functions. The main task of the Landing Gear is to absorb the kinetic energy at touch-down during the landing on the comet. In addition the LG provides a mechanical interface for the anchoring harpoons, which are attached to the LG's central structure and 
should have been fired after landing detection to avoid Philae bouncing after touchdown [17].

\subsection{Philae constraints}

The Philae constraints are mainly related to the touch down condition and the descent duration. They are summarized in the following table and inputs are provided in [2] (Fig. 2 and Table 3).

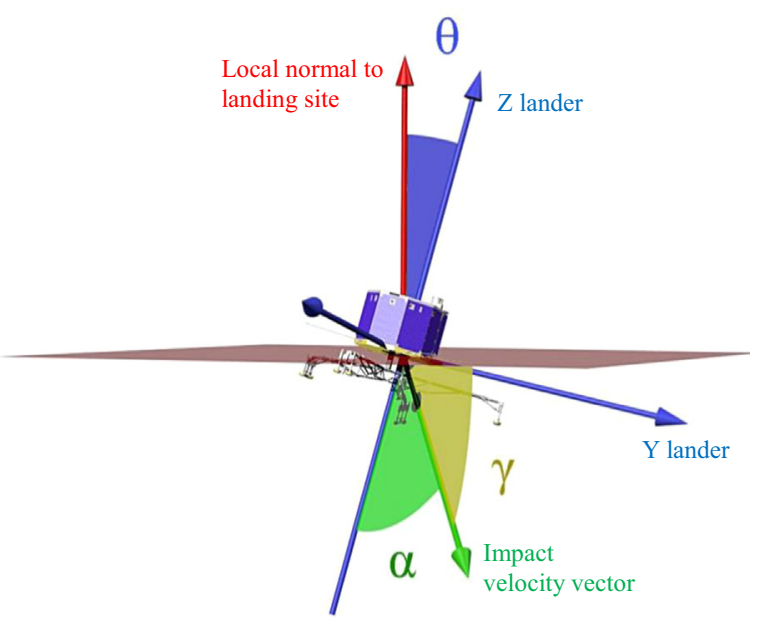

Fig. 2. Description of impact parameters.

Table 3

Philae constraints on descent and impact.

\begin{tabular}{lll}
\hline Constraint & Min & Max \\
\hline Descent duration & $30 \mathrm{~min}$ & $360 \mathrm{~min}$ \\
Attitude angle $=\boldsymbol{\theta}$ & $0 \mathrm{deg}$. & $30 \mathrm{deg}$. \\
Path angle $=\boldsymbol{\gamma}$ & $-90 \mathrm{deg}$. & $-60 \mathrm{deg}$. \\
Attack angle $=\boldsymbol{\alpha}$ & $0 \mathrm{deg}$. & $30 \mathrm{deg}$. \\
Velocity at impact & $0.2 \mathrm{~m} / \mathrm{s}$ & $1.1 \mathrm{~m} / \mathrm{s}$ \\
\hline
\end{tabular}

In order to ensure the proper functioning of Philae for the long Term Science Phase (LTS) some constraints are placed on the illumination conditions of the landing site: the daylight duration at the landing site should be longer than $6.2 \mathrm{~h}$ ( $\sim$ half the rotation period) and shorter than $11.9 \mathrm{~h}$ (leaving at least $30 \mathrm{~min}$ of night).

\subsection{Philae delivery scenario}

Philae was separated from the Rosetta Orbiter with an adjustable ejection device (Mechanical Separation System, MSS), able to deliver a $\Delta V$ impulse ranging from $5 \mathrm{~cm} / \mathrm{s}$ to $50 \mathrm{~cm} / \mathrm{s}$. In case of separation mechanism failure, an emergency spring could also separate the Lander from the Orbiter with a fixed impulse of $18.74 \mathrm{~cm} / \mathrm{s}$, several seconds after the nominal separation time. Philae then descended to the surface of the comet on a ballistic trajectory, stabilized around its $Z$-axis by an internal fly-wheel. At touchdown, harpoons should have anchored Philae to ground and the ADS should have been fired to minimize any possible rebouncing.

The Orbiter trajectory design for pre-landing and delivery phase was under ESOC responsibility. For Philae delivery, the Orbiter trajectory design was to perform a maneuver between 2 and $3 \mathrm{~h}$ before the separation sequence was triggered. This maneuver brought the orbiter from the pre-delivery orbit (elliptic orbit $10 \mathrm{~km} \times 20 \mathrm{~km}$ ) to the separation trajectory. Due to the degrees of freedom introduced by the pre-delivery maneuver, it was then possible to access a large domain of points in the surface of the comet with acceptable conditions. An additional post-delivery maneuver was executed $30 \mathrm{~min}$ after separation (once attitude perturbations due to Lander separation have been damped) to optimize Lander-Orbiter visibility at landing.

Two operational strategies have been defined by RLGS for the Lander separation:

- Option 1 (01) strategy: the separation $\Delta V$ is fixed at the same value as the emergency separation $(0.1874 \mathrm{~m} / \mathrm{s})$. So, in case of an emergency separation, the impact point on the comet surface was the same as the nominal targeted landing point. The main drawback of this strategy is the long descent durations obtained.

- Option 2 (02) strategy: the separation $\Delta V$ can be optimized between $0.3 \mathrm{~m} / \mathrm{s}$ and $0.5 \mathrm{~m} / \mathrm{s}$. In practice, it was always tuned to the maximal value of $50 \mathrm{~cm} / \mathrm{s}$ in order to limit the descent duration. In case of an emergency separation, the actual reached point was very far from the targeted one. Most often the Lander would not even have impacted the comet surface (Fig. 3).

\section{Flight Dynamics analyses for landing site selection process}

The Philae landing site selection process started at the end of July 2014 with the first detailed observations of the comet nucleus. It ended on the 12th of October with the confirmation of the socalled site "J" ( "Agilkia") by RLGS. In the scope of the Landing Site Selection Process, SONC Flight Dynamics [3] team in Toulouse was in charge of computing the technical data for the selection (several

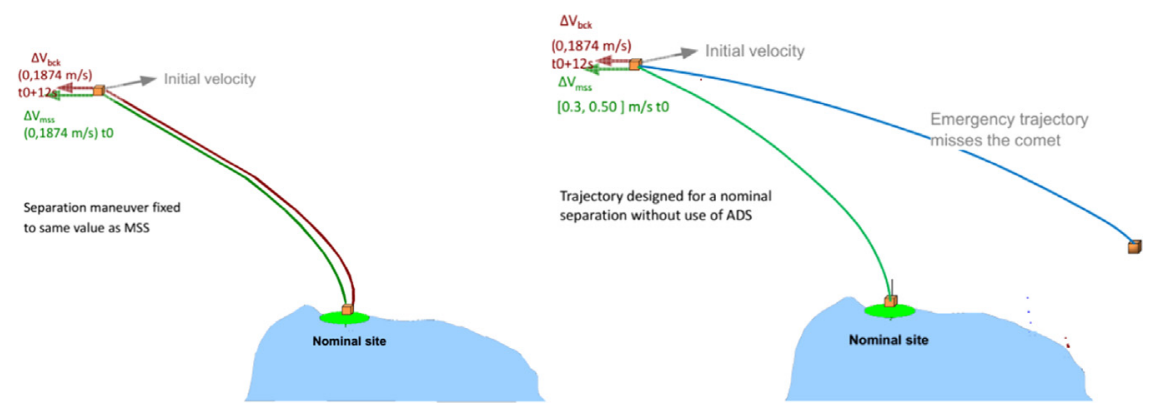

Fig. 3. Lander separation strategies $\mathrm{O} 1$ (left) and $\mathrm{O} 2$ (right). 
versions of mission analysis document $\left.{ }^{[\mathrm{SONC}]}\right)$. At that time, targeted date for Lander delivery was 11th November 2014 and later it was shifted to 12th of November. Several loops of Flight Dynamics analyses have been carried up with improving knowledge of the comet nucleus and coma characteristics to make the down selection of the final landing site (Fig. 1).

\subsection{Preliminary analysis}

In July 2014, the OSIRIS camera on Rosetta showed the first images of the comet nucleus where the global shape was clearly visible. These first pictures were a real surprise since they revealed that the body of comet $67 / \mathrm{C}-\mathrm{G}$ had actually two different lobes (see Fig. 4). The OSIRIS team was able to deliver on the 25th of July the first set of comet model, including a global Digital Terrain Model (DTM) with an approximate horizontal resolution of $500 \mathrm{~m}$. The total volume of the comet was estimated to be $20.28 \mathrm{~km}^{3}$. The comet rotation period was estimated to be $12.4043 \mathrm{~h}$ (from the analysis of light curves obtained between March and July 2014).

The rotation axis associated with this first shape model had a declination of $63.5 \mathrm{deg}$ and a right ascension of $72^{\circ}$ in the EME2000 reference frame. Rosetta was too far away from the comet to allow a mass determination.

Using the shape and the orientation parameters, the illumination conditions on the comet surface for the day of landing (11th November) have been computed. The candidate landing sites had to be chosen in the North hemisphere ( $>$ half rotation illumination) and excluding zones beyond the polar circle of the comet with constant illumination (Fig. 5).
Table 4

Rotation axis parameters $4 / 8 / 2014$.

\begin{tabular}{lll}
\hline Parameter & RMOC & OSIRIS \\
\hline Right Ascension EME2000 (deg.) & 69.473 & 69.370 \\
Declination EME2000 (deg.) & 64.011 & 64.132 \\
Period (h) & 12.4038 & 12.4043 \\
\hline
\end{tabular}

\subsection{Pre-selection of 10 candidate landing sites}

The first official LSSP loop began the 4th of August 2014 with the delivery of the first comet model sets by RMOC and a refined comet model set by OSIRIS (shape 2). At that time, the distance to the comet had reduced drastically and the resolution of the comet models was greatly improved (about $20 \mathrm{~m}$ horizontal resolution).

CNES used both shape models and the comparison of the two showed global consistency. The comet body-fixed frame defined by the RMOC shape model was the reference frame for the navigation studies. So, the OSIRIS team converted the coordinates of the model from the reference frame they used to perform the shape reconstruction to the reference frame used by RMOC. The alignment was performed by minimizing the Hausdorff distance between the two 3D models. There was a translation of about $285 \mathrm{~m}$ between RMOC and OSIRIS reference frames (implying a shift up to several degrees in latitude and longitude for a given point at the comet surface). The orientation parameters were provided by both teams (see Table 4).

Using these models, the illumination conditions at comet
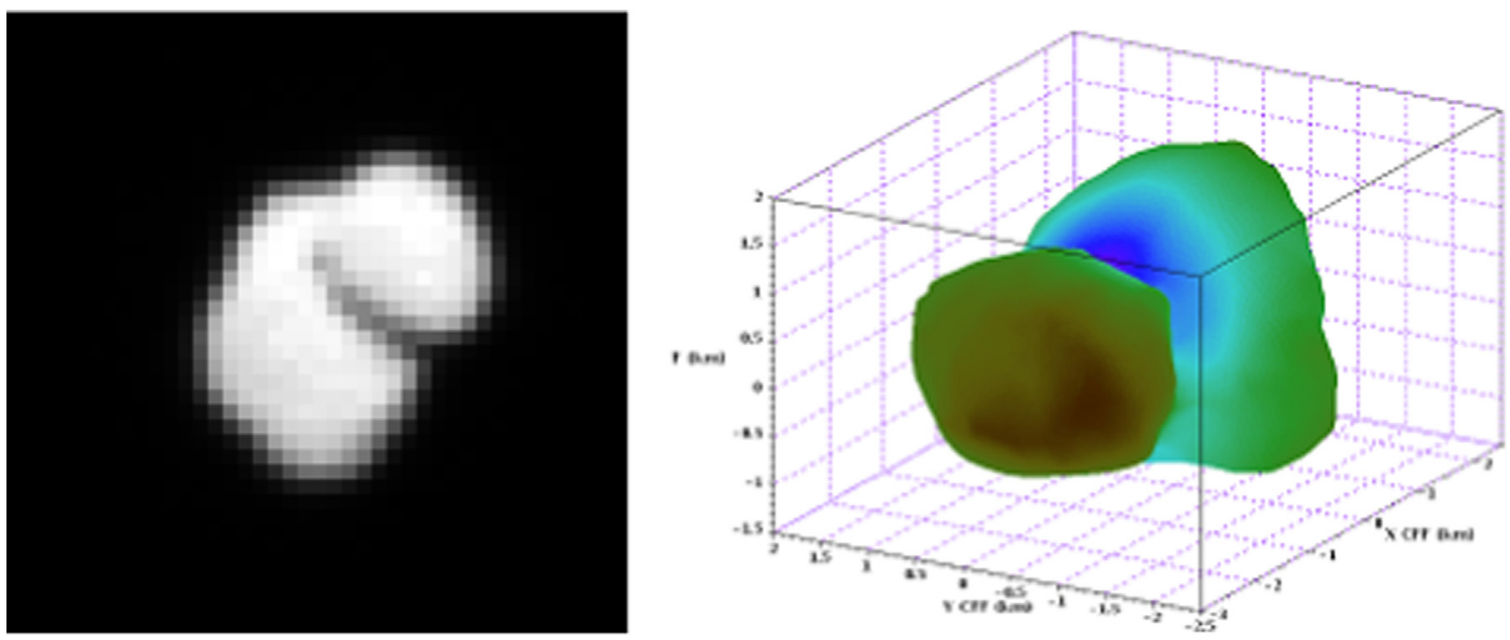

Fig. 4. Comet $67 \mathrm{P} / \mathrm{C}-\mathrm{G}$ as seen by OSIRIS camera on 14 th July 2014 (from a distance of 12,000 km).

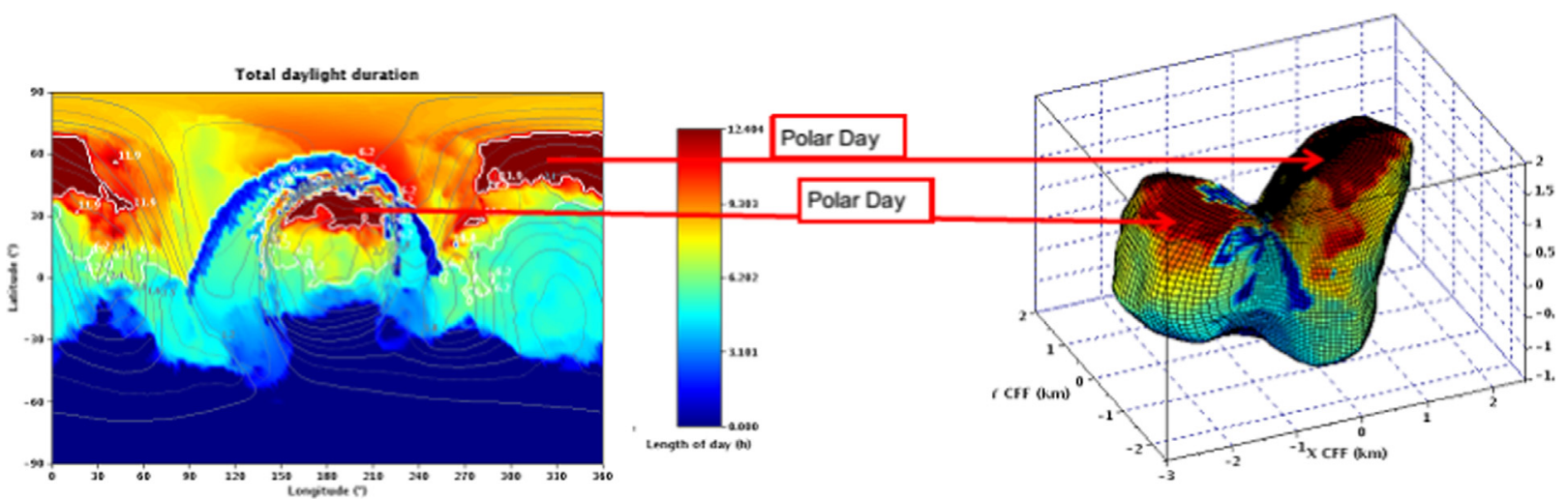

Fig. 5. Illumination map (OSIRIS shape model). 

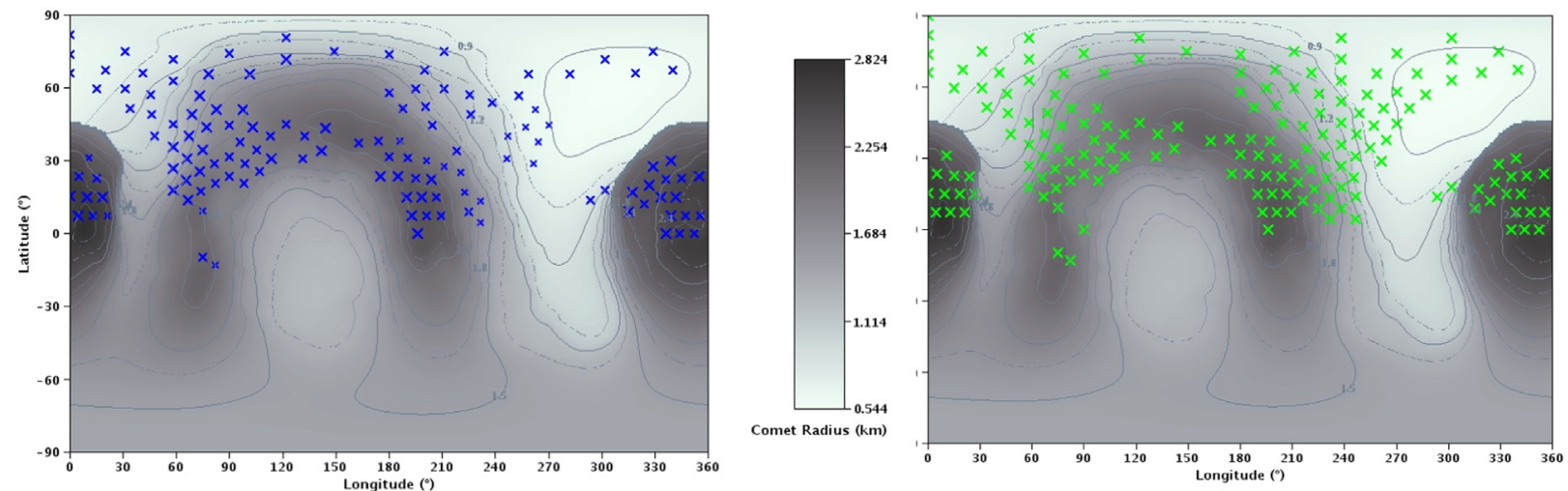

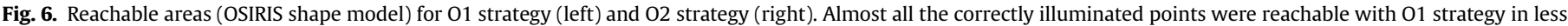

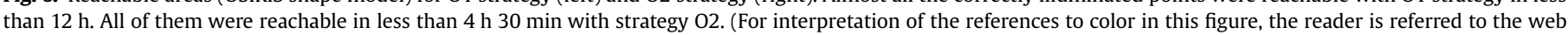
version of this article.)

surface were computed again. The zones where the illumination conditions were acceptable represented only a small part of the comet surface. For all acceptable places in terms of illumination, SONC-FD computed if it was possible to find landing trajectories fulfilling the various mission constraints. As many constraints presented some range of values, the search was performed through a screening of the various parameters. The results are synthetized by the so-called reachable area (Fig. 6). Each colored point on Fig. 6 indicates where Philae can land here without violating a mission constraint.

The computation of landing trajectories requires using some of the comet models presented in Table 2. RMOC estimated from navigation that the nucleus GM is $660 \mathrm{~m}^{3} \mathrm{~s}^{-2}$ but since Rosetta was still too far away from the nucleus, it was not possible to estimate more than the mass and center of mass. As the comet shape is extremely irregular, a mass point model was too approximate and SONC-FD decided to use a simulated gravity field derived from a constant density shape model. The methodology is derived from Werner's work [4]. With this method, using the OSIRIS shape models and mass estimated by RMOC, CNES-GRGS team generated a polyhedron gravity model using the constant density assumption. Comparison of the center of mass position resulting from the constant density assumption with the estimated position from the navigation data showed an offset of about $60 \mathrm{~m}$. This relatively small value confirmed that for this loop the polyhedron gravity field with constant density could be used. In addition, SONC-FD used for the trajectory analyses an outgassing model provided by the LATMOS team using the first data from ROSINA measurements. Outgassing effect is more important around the subsolar point, but it is generally 50 times lower than gravitation force. Due to the distance from the comet at that time, RMOC reported that it was impossible to estimate the drag force acting on the spacecraft from navigation data. For this step, a large amount of computations was performed, due to the possibilities offered to combine the existing models (two shapes, two gravity models and one outgassing model).

The main conclusion reached by SONC-FD at this step was that all the sites with acceptable illumination conditions could be reached by 01-type descent trajectories lasting less than $12 \mathrm{~h}$ and by 02-type trajectories with duration lower than $4 \mathrm{~h} 30 \mathrm{~min}$. When more restrictive constraints on the duration were required, the reachable area was reduced accordingly.

To efficiently select the 5 best landing sites, SONC-FD should analyze the local illumination of Philae and the communication

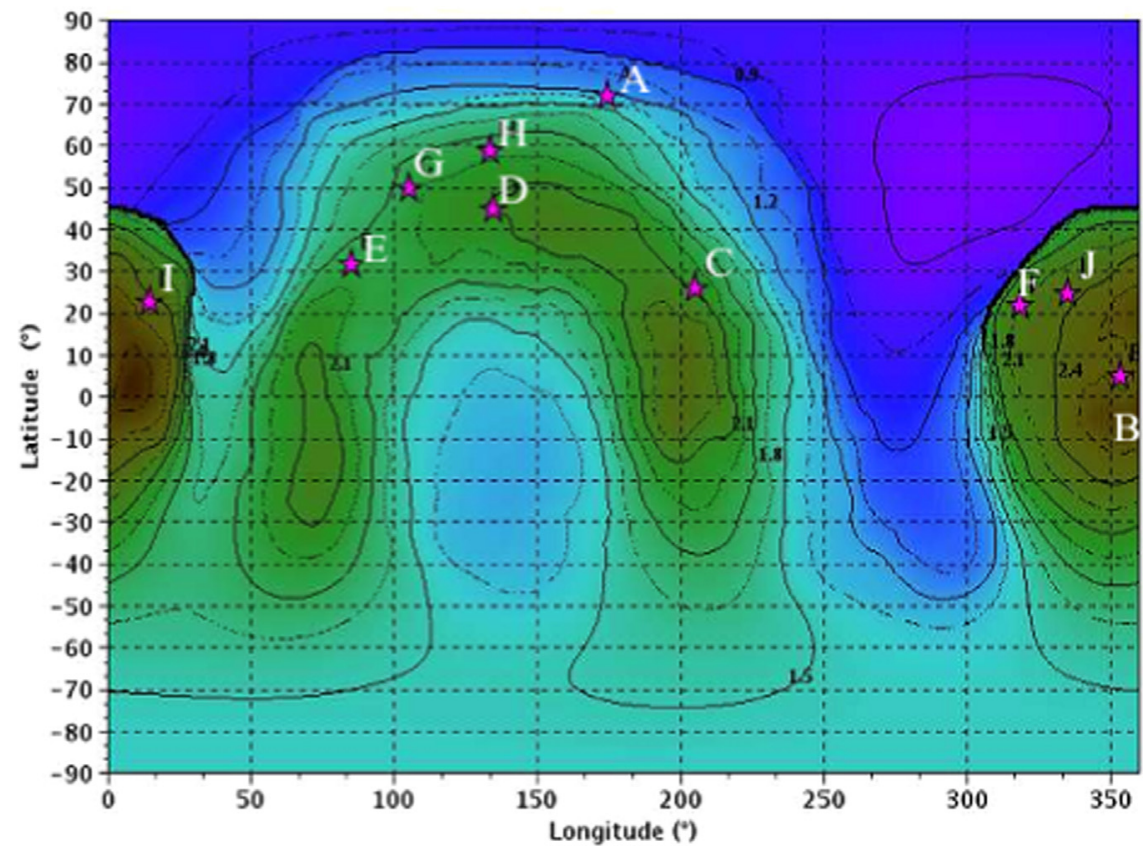

Fig. 7. Pre-selected landing zones 20/08/2014. 
Table 5

Coordinates of 10 sites (OSIRIS frame).

\begin{tabular}{lll}
\hline Name & Latitude & Longitude \\
\hline A & 72 & 174 \\
B & 5.2 & 353 \\
C & 26 & 205 \\
D & 45 & 134 \\
E & 32 & 85 \\
F & 22 & 318 \\
G & 50 & 105 \\
H & 59 & 133 \\
I & 23 & 14 \\
J & 25 & 335
\end{tabular}

Table 6

Coordinates of the 5 candidate sites.

\begin{tabular}{|c|c|c|c|c|}
\hline \multirow[t]{2}{*}{ Site } & \multicolumn{2}{|c|}{ OSIRIS } & \multicolumn{2}{|c|}{ RMOC } \\
\hline & Lat & Long & Lat & Long \\
\hline I & 22 & 10 & 20.2 & 10.2 \\
\hline$C$ & 25 & 203 & 28.8 & 201.4 \\
\hline $\mathrm{J}$ & 15 & 335 & 14.3 & 340.2 \\
\hline A & 72 & 170 & 77.8 & 127.45 \\
\hline B & 5.4 & 351.8 & 5.2 & 355.4 \\
\hline
\end{tabular}

opportunities and LCC should analyze the temperature at landing site and power budget required to realize FSS and LTS. This work required having individual landing sites instead of landing areas. Then a LSSP meeting was held on the 20th August 2014 to define 10 possible candidate landing sites distributed over the reachable areas. The selection was made based exclusively on technical criteria, and without taking into account scientific interest of the landing site. Analyses done before the beginning of the operations had shown that the landing ellipse could be as big as $1 \mathrm{~km}^{2}$. Taking into account this uncertainty, almost the whole reachable zone on the comet was covered by these 10 sites, named from A to J (Fig. 7).

\subsection{Shortlist of 5 candidate sites}

Between the 20th and the 24th of August, a dedicated analysis was performed for each of the 10 landing sites (slope analysis,
OSIRIS dedicated images around the sites, communication opportunities during LTS phase...). A two-days meeting was held in CNES Toulouse on 23rd and 24th August during which these technical criteria were presented. The scientific interest of the landing sites was also considered and the list was reduced to 5 candidate landing sites: I, C, J, A and B. The coordinates were also fine-tuned in order to place them in the center of the flat area of the landing zones (slope analysis in the whole area taking into account pre-defined uncertainty on landing point) (Tables 5 and 6).

On the 24th August, RLGS provided RMOC with the list of the 5 candidate landing sites. 15 days later, RMOC sent back the operational feasibility analysis and the corresponding descent trajectories for the two pre-defined scenario $\mathrm{O} 1$ and $\mathrm{O} 2$.

\subsection{Selection of nominal and back-up sites}

Four of the five shortlisted sites were considered reachable by RMOC. Site A was rejected as it was located close to the junction of the two comet lobes: on one hand the gravity model was poorly accurate for the junction zone and on the other hand the outgassing was important around this zone. So flying over or at the vicinity of the zone was too risky.

For site $\mathrm{C}$, the $\mathrm{O} 1$ strategy was discarded because the touchdown would have occurred too early in the day with low sun illumination and consequently bad imaging conditions. For this site, the $\mathrm{O} 2$ strategy was preferred. For remaining sites, both $\mathrm{O} 1$ and $\mathrm{O} 2$ strategies were acceptable.

At the beginning of September 2014, Rosetta was at a distance of about $30 \mathrm{~km}$ from the center of the comet nucleus. A new global DTM (with a resolution of about $10 \mathrm{~m}$ ) was provided by RMOC using latest NAVCAM and OSIRIS images. OSIRIS provided local DTMs of the candidate landing areas with resolution of a few meters. Both teams provided updated rotation parameters of the comet. The GM value estimated from navigation data $\left(660 \mathrm{~m}^{3} \mathrm{~s}^{-2}\right)$ did not change. Depending on the shape model, this corresponded to a density of $466.1 \mathrm{~kg} \mathrm{~m}^{-3}$ with RMOC model and $471.2 \mathrm{~kg} \mathrm{~m}^{-3}$ OSIRIS model. LATMOS team updated the outgassing model based on the latest ROSINA measurements.

The outgassing effect was still 10 to 50 times lower than the gravitational force. RMOC was still not able to estimate outgassing effect from navigation data.
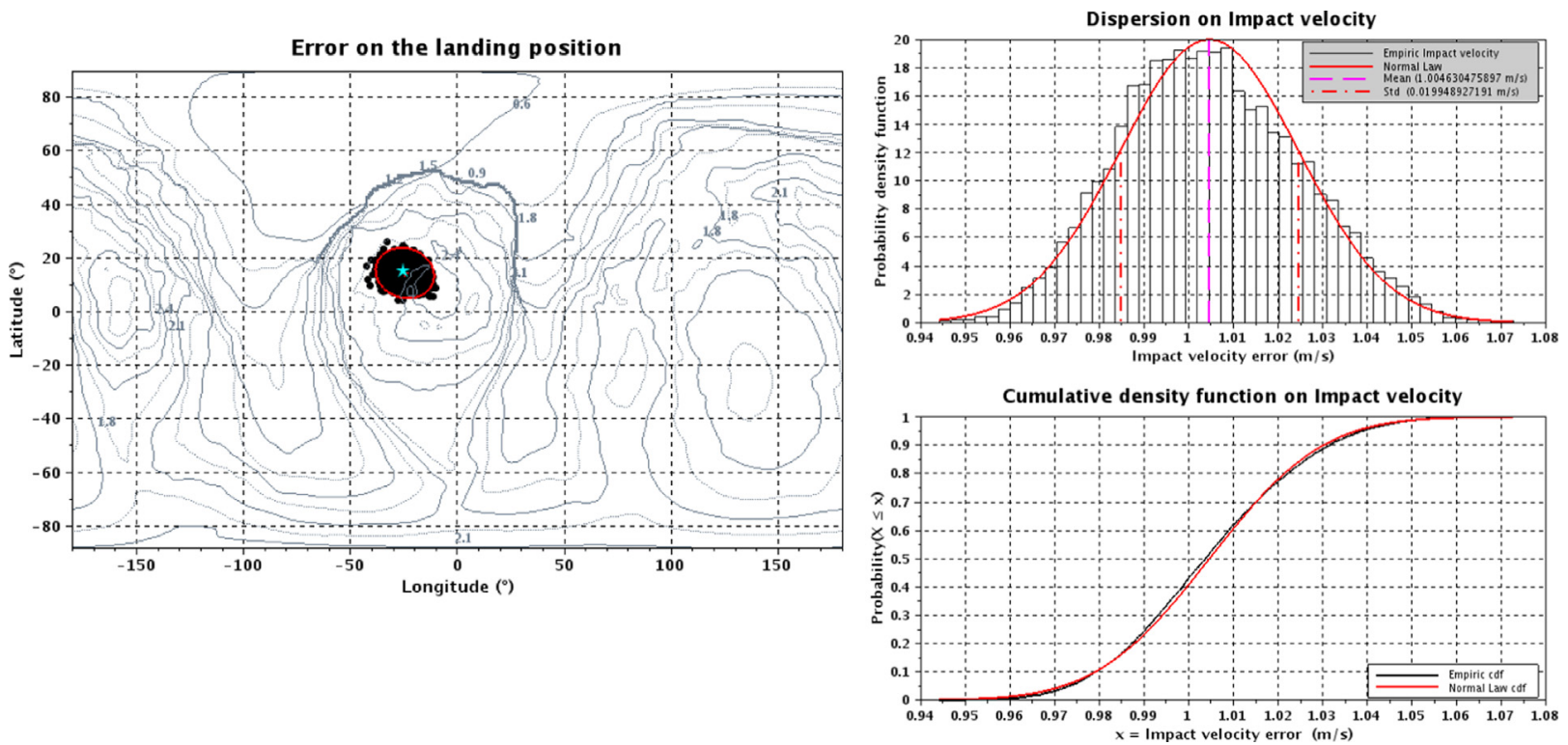

Fig. 8. Dispersion analysis site J (left dispersion ellipse, right dispersion on impact velocity). 

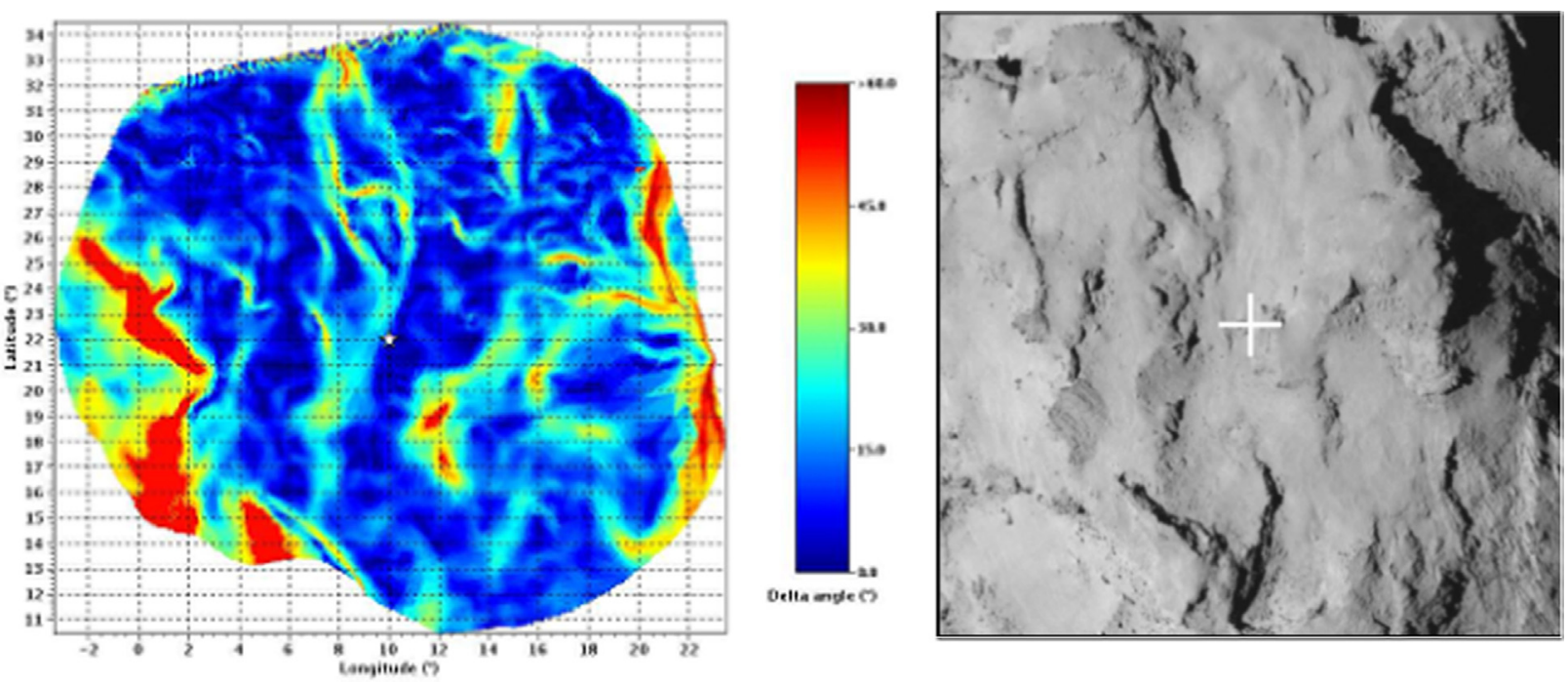

Fig. 9. Slope deviation angle computed from DTM (left) OSIRIS images for site I (right, distance $30 \mathrm{~km}$ ).

Taking into account these inputs, a dispersion analysis base on Monte-Carlo method was performed for each of the trajectories computed by SONC-FD.

The objective of this dispersion analysis was to derive the dispersion ellipse and to check what percentage of trajectories fulfilled the Orbiter and Lander constraints. For instance, for site J and 01 strategy, the dispersion ellipse and the statistical distribution of impact velocity have been derived from $10^{4}$ simulations (Fig. 8). The resulting landing ellipse covered a large area $(500 \mathrm{~m} \times 400 \mathrm{~m})$ and the maximum limit of impact velocity at $1.1 \mathrm{~m} / \mathrm{s}$ was never exceeded. The main contribution to the dispersion was the uncertainty on the Orbiter position at separation propagated over the $7 \mathrm{~h}$ of descent. Dispersion on the touchdown time was estimated around \pm 20 min.

The statistical distribution of the slopes in the landing area was also a criterion for the ranking. To ensure a safe landing, the angle between Lander $Z$ axis and the local normal had to be lower than $30^{\circ}$. The mean deviation of the local normal w.r.t. the targeted normal, so called "slope deviation angle", higher than $25^{\circ}$ were not desirable. None of the 5 landing sites was $100 \%$ safe from this point of view. In terms of flatness, the two extreme sites were site B and I. Site B was the flattest with $85 \%$ of landing area with slopes deviation angle lower than $25^{\circ}$. For site I, which was the roughest one only $70 \%$ of the area showed slope deviation angles lower than $25^{\circ}$. High resolution images provided by OSIRIS team were also used as a visual confirmation of the slope analysis (Fig. 9).

The illumination conditions around landing sites were also studied in detail using the updated shape models provided by OSIRIS and RMOC teams. The covered period was from landing up to 6 months after landing (potential end of LTS). Indeed, at least $6.2 \mathrm{~h}$ of illumination are necessary to recharge the battery.

For site J, the illumination conditions were globally acceptable in the whole area: $85 \%$ of the landing area had more than $6.2 \mathrm{~h}$ of daylight duration just after landing but 60\% did not respect the $6.2 \mathrm{~h}$ minimum duration 6 months after landing (corresponding to FSS).

Finally, on 13th and 14th September a second LSSP meeting was held in CNES Toulouse to decide the final ranking based on technical results and scientific interest. Site J was finally chosen as
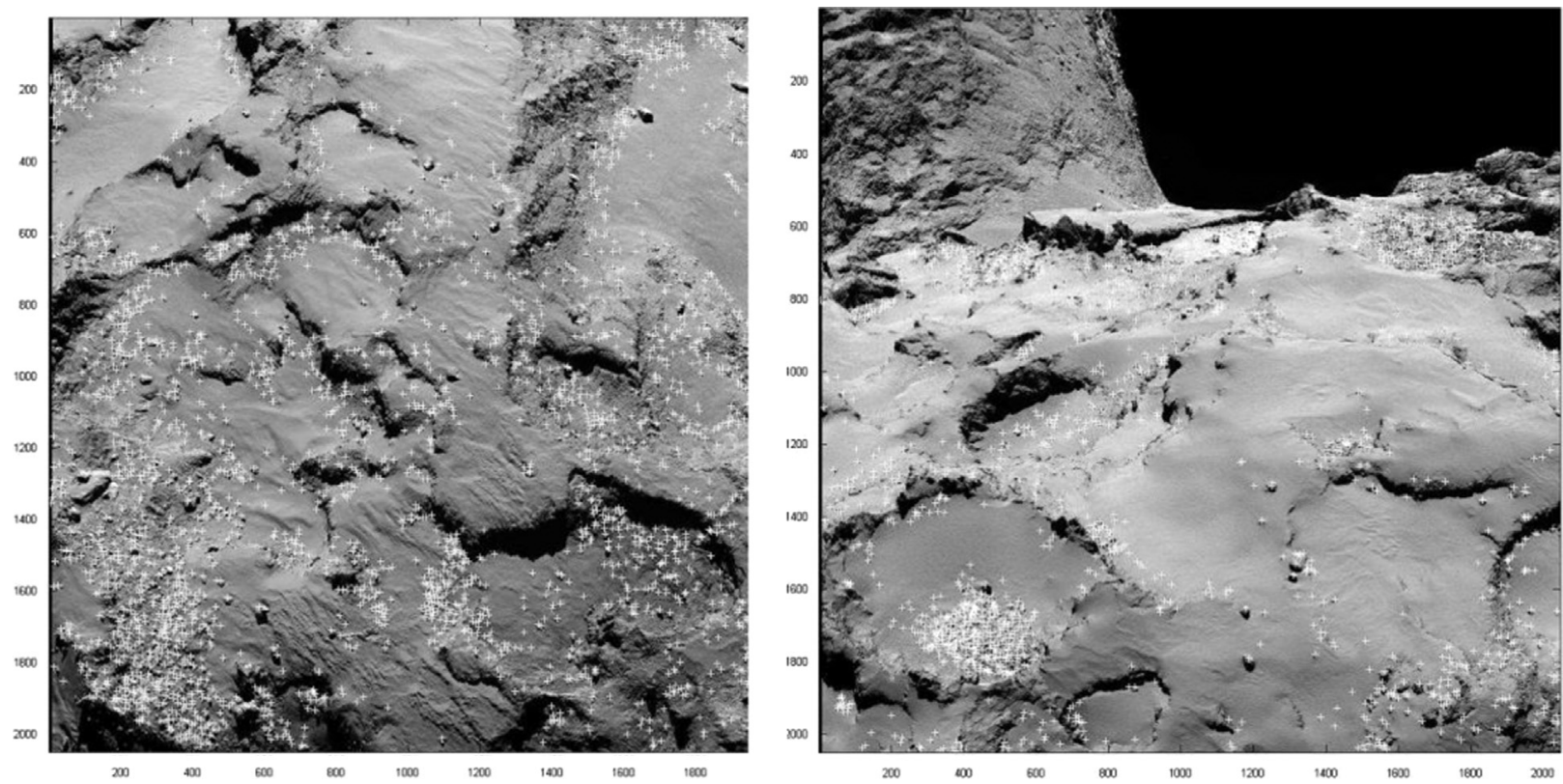

Fig. 10. Position of the boulders inside landing area J (left) and C (right) from OSIRIS team. 
the nominal landing site (using 01 strategy) and site $\mathrm{C}$ as the backup landing site (using $\mathrm{O} 2$ strategy).

\subsection{Confirmation of nominal landing site and complementary analyses}

On 14th September, site J was thus chosen as the nominal landing site. Following a competition initiated by ESA, CNES, DLR and ASI (the Italian space agency), the site was named "Agilkia" after the Nile island where the ancient temple of Philae have been moved to be rescued after the construction of the Aswan dam. At the end of September, in the scope of the preparations of the final operational trajectory for Rosetta, RMOC announced that Lander delivery would occur on 12th November 2014 instead of 11th November as stated before.

At the beginning of October, Rosetta came closer to the comet, up to $10 \mathrm{~km}$ from the center thanks to the low outgassing activity. All necessary models (shape, gravity, outgassing...) were updated with the latest available data. OSIRIS also provided local DTM for the nominal and back-up sites. RMOC provided a new gravity model, with an updated GM of $667 \mathrm{~m}^{3} \mathrm{~s}^{-2}$ and spherical harmonic coefficients up to the degree 3. CNES-GRGS derived from the OSIRIS shape model a polyhedron gravity model with the constant density assumption. The offset of the center of mass w.r.t. the spherical model was about $50 \mathrm{~m}$, which indicated that the constant density estimation was still valid for trajectory extrapolation. LATMOS and RMOC provided outgassing models. Both models indicated that the outgassing force was negligible, at least 50 times lower, than the gravity force.

RMOC delivered the final operational trajectory. The separation was planned on 12 th November at $08 \mathrm{~h} 35 \mathrm{m00s}$ UT at $22.5 \mathrm{~km}$ from the center of the comet and the expected touchdown time was $15 \mathrm{~h} 34 \mathrm{~min} 55 \mathrm{~s}$ UT with an impact velocity of $0.95 \mathrm{~m} / \mathrm{s}$.

At this step, thanks to the low altitude of Rosetta, OSIRIS was able to provide detailed statistics and localization on the boulders inside the landing area (position, sizes for boulder larger than $1 \mathrm{~m}$ and statistical distribution extrapolated up to $10 \mathrm{~cm}$ boulders).

SONC-FD computed the risk to land on a boulder inside the landing ellipse. Each boulder was represented by a disk. Its radius was the summation of the radius of the boulder and a safety radius slightly larger than Philae. Using the Monte Carlo trials, the probability was derived by counting the number of landing points inside a disk over the total number of trials (Fig. 10).

The estimated probability to land in a place free of boulders was $82 \%$ for site J and $90 \%$ for site C (Fig. 11). The probability estimation is approximate and one can consider these two results are close.

The LSSP committee took into account all provided parameters and it clearly appears that site J was a better choice than site C.

As a consequence, a final LSSP meeting on 10th of October 2014 decided to confirm the choice of site $\mathrm{J}$ as the nominal landing site.

On 14th October 2014, the Lander Operational Readiness Review gave the final GO for the beginning of the landing operations.

\section{Separation, descent and landing}

On 12th November 2014 at $08 \mathrm{~h} 35 \mathrm{~m}$ UT, the Lander finally separated from Rosetta as planned. The pre-delivery maneuver was perfectly executed and the MSS provided a very accurate separation $\Delta V$ (reporting a $\Delta V$ value of $18.76 \mathrm{~cm} / \mathrm{s}$ instead of the expected $18.74 \mathrm{~cm} /$ s). Several images of the Lander during descent were provided by OSIRIS and seemed to indicate that the trajectory was probably close to nominal. An image of Rosetta by the CIVA camera of the Lander was taken several minutes after separation. The image was coherent with estimation performed during the preparation of operations, even if Rosetta was in the border of CIVA field of view, which indicated a rather high rotation rate of the Lander around its $Z$ axis. According the measurement realized by CONSERT radar and ROMAP

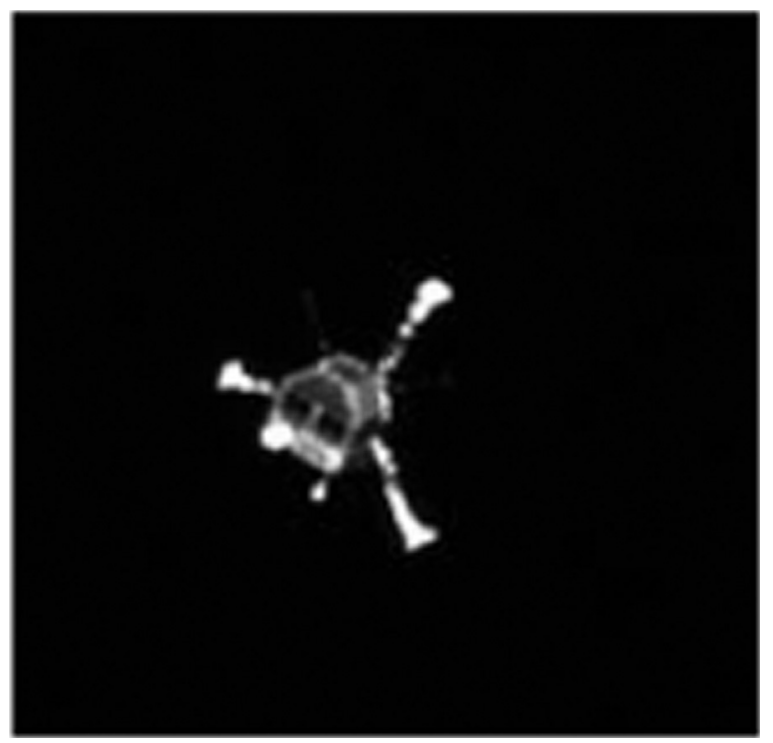

Fig. 12. Philae seen by OSIRIS during descent.

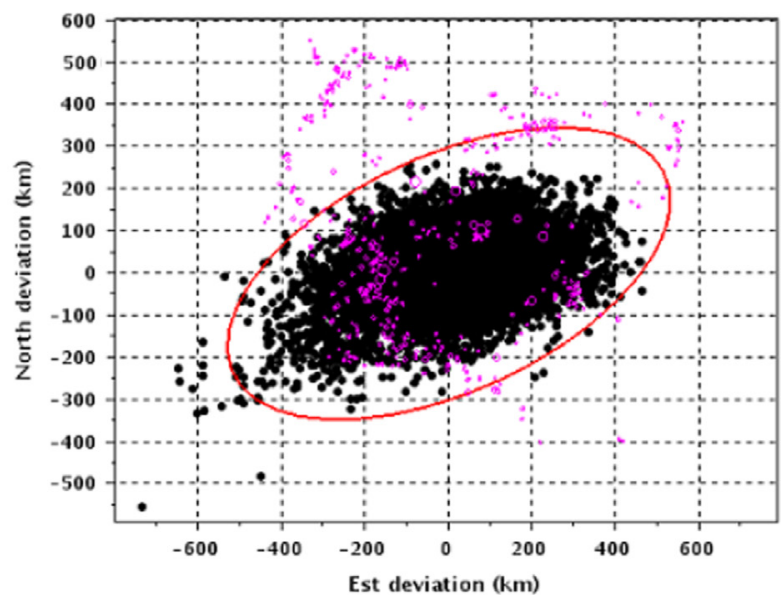

Fig. 11. Statistical distribution of boulders inside landing ellipse for site J (left) and C (right). 


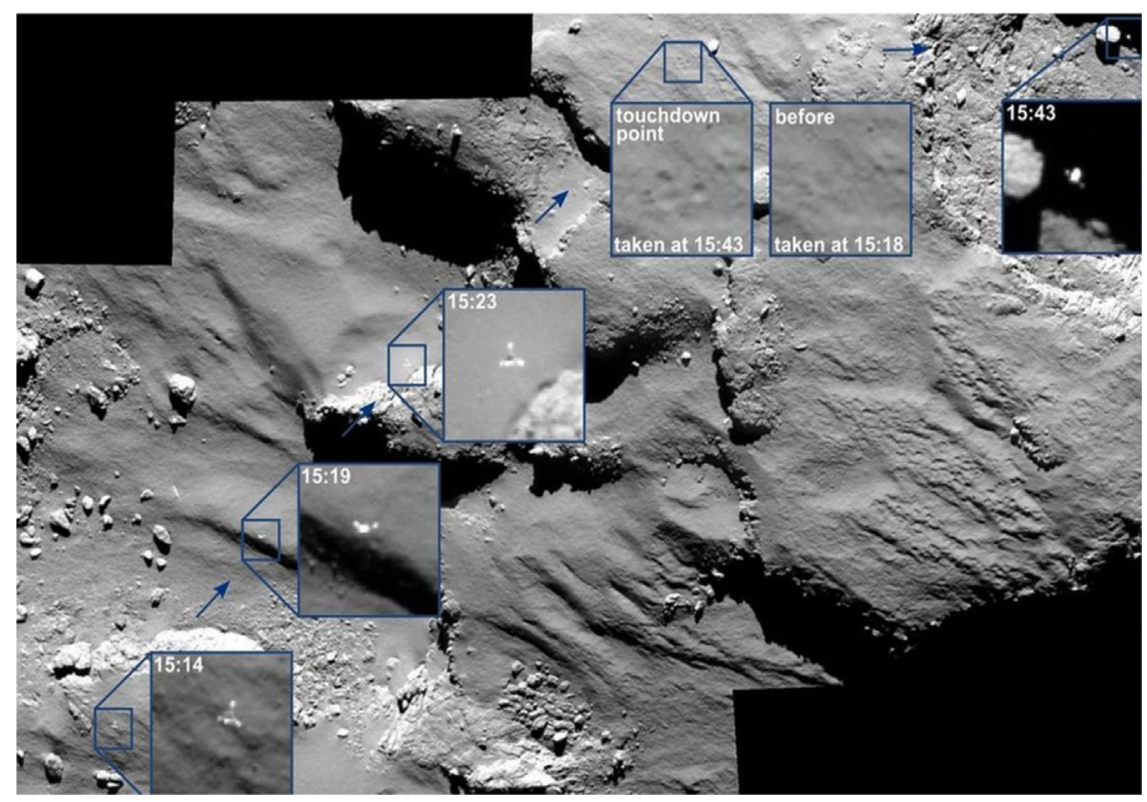

Fig. 13. Philae just before and after touch-down.

magnetometer, the rotation rate was estimated to one rotation every 8.6 min (Figs. 12 and 13).

The touch-down signal on the surface of the comet was registered $50 \mathrm{~s}$ earlier than expected and at a distance of less than $120 \mathrm{~m}$ from the nominal target site. Unfortunately, due to a double failure of the harpoons and the thruster (that should have performed a DV to push Philae to the ground), the lander bounced for $1 \mathrm{~h} 50 \mathrm{~min}$ after first touch-down. Several collisions with the ground were identified and Philae finally stopped at a site approximately $1 \mathrm{~km}$ away from Agilkia. Despite this complicated landing, Philae communicated easily with Rosetta, more than $2 \mathrm{~h}$ per comet rotation and performed its First Science Sequence operations. All Philae instruments were activated during the following 5 comet days $(57 \mathrm{~h})$. Unfortunately, it became obvious that the Lander was in a poorly illuminated area, and that recharging the secondary battery was not possible at this time. On the 15th November at 22 h $30 \mathrm{~min}$, contact was lost with Philae. Hopefully, Philae landed at latitudes South to Agilkia. It meant that the landing site would benefit from longer illumination period in the month to come. Moreover the solar flux would increase as the comet would get closer to the sun. Philae actually booted again at the end of April or early May, and a first contact was re-established on the 13 th June.

\section{Position and attitude of Philae on its final landing site}

As soon as Philae landed, several teams (CNES robotics team, ROMAP, CONSERT, SONC FD...) worked simultaneously on the determination of position and attitude of the lander on its final landing site [7]. SONC-FD team was in charge of validating the results for Lander operations. Thanks to Lander contacts in June and July, additional data was received that helped to refine the analyses done after landing.

\subsection{Attitude determination}

SONC-FD and ROMAP teams worked together on the attitude determination of Philae. The methods used by both team did not require knowing Philae's position to determine the attitude of Philae so it was carried on independently from the search of Philae final landing site. These methods provide the orientation of the lander frame (LDR, cf. Fig. 14) with respect to the Comet Fixed Frame (CFF, cf. Fig. 14). CFF origin is located at the center of mass of the comet and its $Z$ axis is aligned with the comet rotation axis. This frame rotates with the comet (period circa $12.4 \mathrm{~h}$ ).

The ROMAP instrument team was the first to be able to determine the full attitude (method is described in [8]).

SONC-FD teams used the currents generated by the solar arrays. At first order, the current produced by a solar array is proportional to the cosine of the incidence angle (angle between the normal to a solar array and the direction of the sun).

Some basic algebraic manipulations show that:

- The sun azimuth expressed in the LDR frame is determined from the currents output from two lateral walls.

- The sun declination is determined from currents output from the lid and a lateral wall.

One then compute the sun direction for several dates, and as the comet rotation is well characterized, one can determine the attitude of the lander with respect to the comet fixed frame. As explained, it is not necessary to know Philae's position. The attitude estimation is performed using a least square approach tailored to quaternion [16].

The estimation quality depends on:

- The shadowing of the solar array. A few shaded cells may lead to switch off a string, half of the solar array. This is of course impacting the quality of attitude determination.

- The width of the time span used for the attitude determination: if this time span is too short, the sun direction is not changing significantly in the lander frame and the determined attitude will not be very accurate.

- The dust accumulation of the solar arrays: it modifies the efficiency of each solar array and introduces systematic bias.

During the FSS, the wall 2, 3 and the lid were poorly illuminated. The estimated attitude was not consistent with the one derived from ROMAP measurements.

In addition, CNES teams also analyzed the pictures taken by CIVA-P camera and were able to derive the sun direction at the 

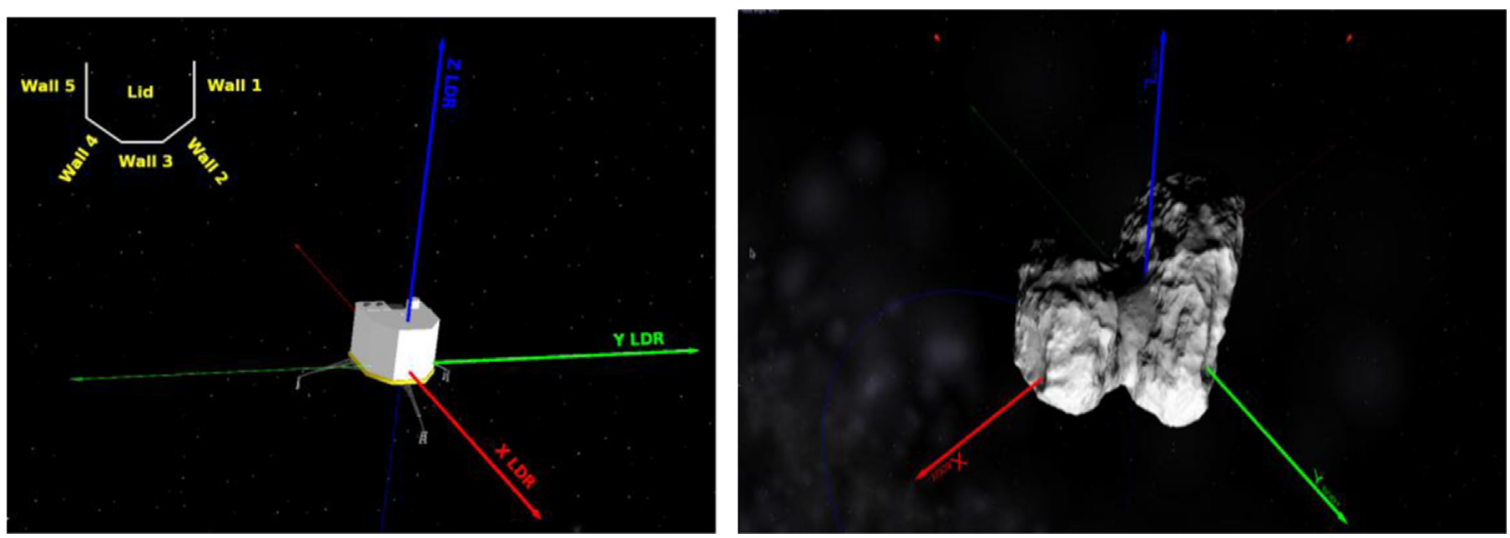

Fig. 14. LDR frame (left) and CFF (right).

date of the acquisition of the image from the shadow of one of Philae's feet. The direction was consistent with the one derived from the ROMAP results. So it was decided to consider ROMAP attitude as correct.

After Philae's wake-up, SONC-FD was able to determine the attitude from the new transmitted HK data but only with data of the 13th of June (cf. Fig. 15). Actually, each time Philae falls asleep, it loses its internal clocks but increments a reboot counter. Therefore all subsequent HK data timestamps are meaningless. Nevertheless, assuming one increment per comet rotation leads to a rough estimate of the day of recording. Of course if for any reason, the lander falls asleep several times per rotation, this date will be wrong. Moreover, this date is approximate as only the day is known but not the period of the day. On the contrary, live HK data is well dated because Rosetta is time stamping received HK data.

HK data of the 13th of June is correctly dated and Philae is sufficiently illuminated (wall 1, 2, 3 and lid producing currents). Wall 1 current is discarded as the output power reaches the saturation limit. As seen, the current produced by this wall is almost constant for $3 \mathrm{~h}$. This means that, this current is not proportional anymore to the cosine of the incidence angle.

The attitude was determined using 70 min of HK with currents from wall 2, 3 and the lid.

As seen on Fig. 15, Walls 2 and 3 present some strong variations. They are probably caused by shadow of surrounding rocks. This is of course impacting the quality of the attitude determination
ROMAP and SONC-FD attitude are rather close: The angle between the two $Z$ LDR axes is $5.5^{\circ}$. The Euler angle $(X, Y, Z)$ between ROMAP attitude and SONC-FD attitude are $\left[1.9^{\circ}, 2.6^{\circ},-14.2^{\circ}\right]$.

Fig. 16 presents a comparison between the HK currents and a rough estimate of the current determined from ROMAP attitude and SONC-FD attitude. By rough estimate, we mean that we just compute the cosine of the incidence angle multiplied by a constant. LCC is doing more accurate current estimation but SONC-FD does not use their model. Nevertheless Fig. 16 clearly shows that, for the 13th of June both predictions are correct. ROMAP seems more accurate than SONC-FD. SONC-FD is prognosticating sunrise on wall 3 half an hour earlier.

\subsection{Position determination}

During the FSS, CONSERT, based on the analysis of ranging measurement between Rosetta and Philae, proposed a first landing area with size of $30 \mathrm{~m} \times 350 \mathrm{~m}$. Later, the team reduced the area to a box $15 \mathrm{~m} \times 150 \mathrm{~m}$ (published in [9]), and finally $22.5 \mathrm{~m} \times 106.5 \mathrm{~m}$ with a most probable area of $22.6 \mathrm{~m} \times 41.5 \mathrm{~m}$ [10].

At the same time, SONC-FD was searching landing areas complying with the estimated sun set and sun rise date estimated from HK data and the acquisition and loss dates of RF links (FSS data).

The OSIRIS and CNES teams analyzed pictures of the comet taken by the Rosetta OSIRIS camera. The process was to compare pictures of the same area taken before and after the landing, if possible with equivalent illumination conditions. Most of the

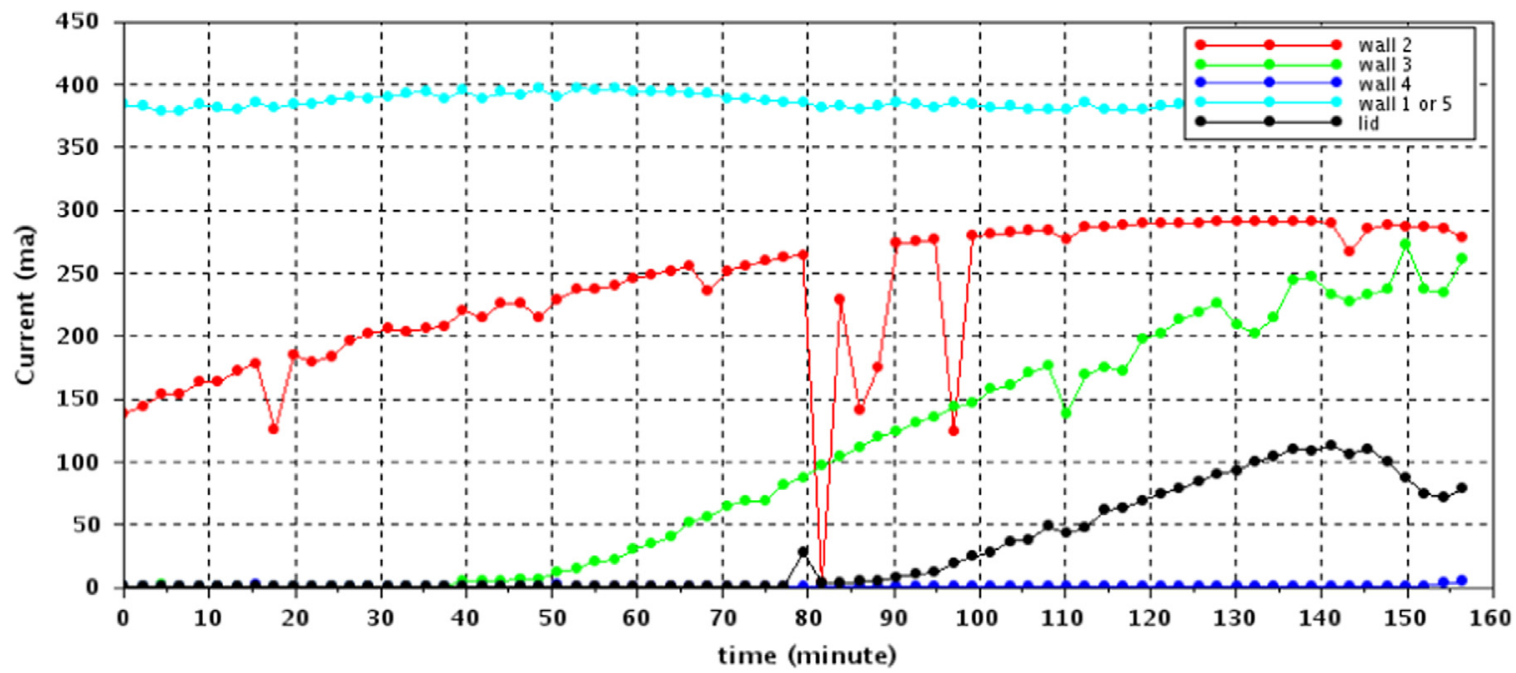

Fig. 15. Currents produced by solar arrays (13th of June time $=0$ is $2015 / 06 / 13$ 21:34:46) 


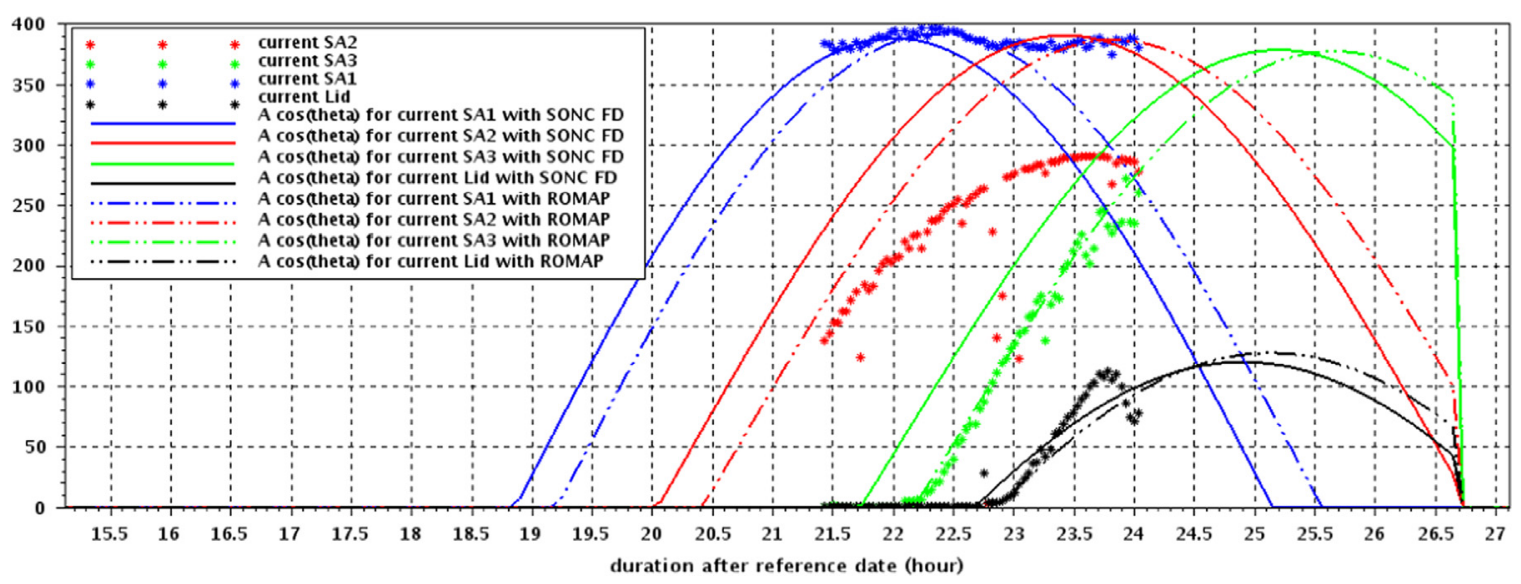

Fig. 16. current output of the solar arrays. (HK, ROMAP, SONC-FD) for the 13th of June 2015.

pictures used for the comparison were taken at $40 \mathrm{~km}$ altitude. As the lander has a metric size, it should be only a few pixels on these images. In this way, the involved teams discovered a few tens of "bright area" that were absent in earlier pictures.

The comet surface is active and due to its outgassing activities, its surface is changing with time with fresh ice, very bright, when exposed to the Sun light.

So SONC-FD developed a process to try to eliminate candidate landing sites.

The idea was to produce exclusion zones. It is obviously hard to know where Philae is but it is quite easy to map places where it cannot be.

The realization of such a map is possible thanks to:

- The illumination period of the lander. The HK voltages and currents indicate when Philae's solar arrays are illuminated.

- The communication windows period between Rosetta/Philae.

The process is the following:

1. We extract an area to analyze from a Digital Terrain Model (DTM). The DTM consists of polyhedron with triangular faces (typical ridge is $5 \mathrm{~m}$ long). We extract triangles located in a $200 \mathrm{~m}$ radius circle around the candidate site from the DTM.

2. For each center of triangle, we determine if the triangle is illumined during the daytime observed during FSS. If not, the candidate landing site may not be located on the triangle. This triangle is excluded as the landing site may not be located here.

3. For the remaining triangles, considering Philae's attitude proposed by ROMAP, we check whether any of the individual solar arrays is illuminated as observed during FSS. If not, the triangle is excluded.

4. For the remaining triangles, we compute if the communication between Philae and Rosetta are possible during the real communication period of FSS.

This process is very easy to implement and has a lot of advantages. At each level of the process, one produces an exclusion map based on a given data.

Map of step 2 are considered very reliable and the largest error sources are the Digital Terrain Models. Maps of step 3, 4 required to trust the Philae attitude provided by ROMAP. Nevertheless, they are rather close to the map of step 2, so results are convergent.

Fig. 17 represents a typical exclusion map. The landing site may be located only in a place without a gray or black dot (north of the map). The yellow, respectively orange, stars delimit the CONSERT possible zone and most likely zone [10].

The red star (longitude, latitude $)=(-1.8,-8.1)$ is the position

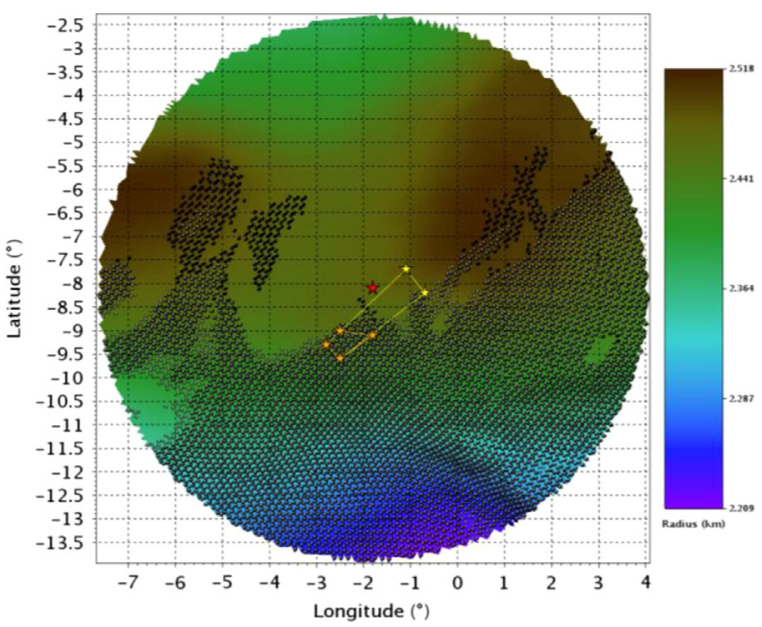

Fig. 17. Exclusion map. (Black dots are not compatible with illumination and gray dot with RF links of the FSS. Yellow and orange stars are the CONSERT possible and most like area, blue star is the SONC-FD position). (For interpretation of the references to color in this figure legend, the reader is referred to the web version of this article.)

Table 7

Dates of acquisition and loss of signal during FSS.

\begin{tabular}{|c|c|c|c|}
\hline $\begin{array}{l}\text { Date of Acquisition } \\
\text { of signal (UTC) }\end{array}$ & $\begin{array}{l}\text { Error in the pre- } \\
\text { diction (min) }\end{array}$ & $\begin{array}{l}\text { Date of loss } \\
\text { of signal } \\
\text { (UTC) }\end{array}$ & $\begin{array}{l}\text { Error in the pre- } \\
\text { diction (min) }\end{array}$ \\
\hline $\begin{array}{l}2014 / 11 / 13-05 \mathrm{~h} \\
32 \mathrm{~min}\end{array}$ & -129 & $\begin{array}{l}2014 / 11 / 13- \\
09 \text { h } 30 \text { min }\end{array}$ & 17 \\
\hline $\begin{array}{l}2014 / 11 / 13-19 \mathrm{~h} \\
27 \mathrm{~min}\end{array}$ & -148 & $\begin{array}{l}2014 / 11 / 13- \\
23 \text { h } 09 \text { min }\end{array}$ & 11 \\
\hline $\begin{array}{l}2014 / 11 / 14-09 \mathrm{~h} \\
00 \mathrm{~min}\end{array}$ & -100 & $\begin{array}{l}2014 / 11 / 14- \\
11 \mathrm{~h} 48 \mathrm{~min}\end{array}$ & 9 \\
\hline $\begin{array}{l}2014 / 11 / 14-21 \mathrm{~h} \\
\quad 47 \mathrm{~min}\end{array}$ & -65 & $\begin{array}{l}2014 / 11 / 15- \\
00 \text { h } 09 \text { min }\end{array}$ & 0 \\
\hline
\end{tabular}

chosen by SONC-FD to perform the communication opportunities computation.

The SONC-FD site is $8 \mathrm{~m}$ away from the possible CONSERT zone (yellow stars) and $46 \mathrm{~m}$ from the most likely zone (orange stars). The most likely zone seems not to be compatible with illumination and RF link but it is close to an admissible zone. So maybe a DTM with a higher resolution may change the results.

The SONC-FD site was chosen in March 2015 and since that choice a better DTM was released by OSIRIS and delivered in July 2015. With the previous DTM (October 2014), the exclusion map was excluded a large part of CONSERT zone. CONSERT team also 


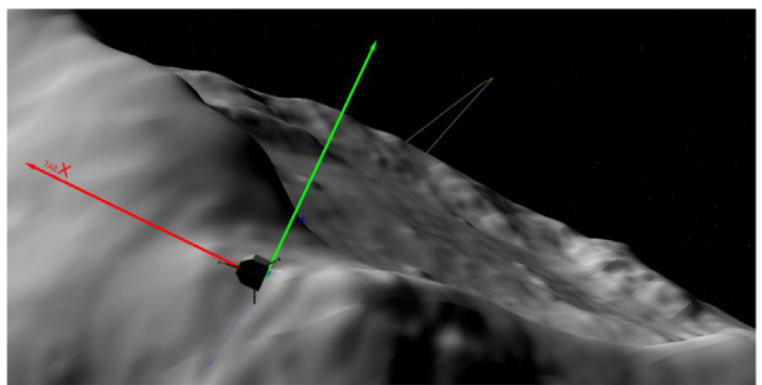

Fig. 18. Philae on its final landing site (Philae size is magnified 25 times).

continued to refine its analysis. It could explain why SONC-FD site is not within the possible CONSERT zones.

With this site, the prediction of sunrise/sunset and RF links were rather coherent with the observations. Table 7 presents the dates of acquisition and loss of signal during FSS. It also contains the error (real date - prediction date) realized when computing the RF link with the ROMAP attitude and considering a $60^{\circ}$ halflobe. Real acquisition occurred between $1 \mathrm{~h}$ and $2 \mathrm{~h}$ earlier than predicted. SONC-FD tools does not compute signal outside the $60^{\circ}$ half-lobe antenna. Of course communications may be possible at larger angles. It seems that we received signal up to $80^{\circ}$ from the $Z$ lander direction. The attitude error of Philae is also responsible of the observed discrepancy.

On the other hand, the predicted loss of signal is rather accurate. Effective loss occurred between 0 and 17 min later than scheduled. This error is created mainly by occultation from high-resolution topography not captured in the OSIRIS DTMs and in a less important way by the inaccuracy on the Philae attitude and position.

Among the candidate sites obtained by means of image analysis, only one was consistent with the exclusion zone. It was discovered by the LAM team [11], (Laboratoire d'Astrophysique de Marseille France). This candidate was only a tens of meters away from the site proposed by SONC-FD based on CONSERT zone.
Later, the LAM team also realized a high resolution local DTM. Using the SONC tools, DTM, position and attitude of Philae, simulated images corresponding to the real images obtained by CIVA-P were generated. The result is rather consistent with real pictures. Such a simulation is not an absolute proof, but it brings confidence in the location.

This landing site is within the CONSERT zone and it is compliant with the illumination conditions and communication windows. Analysis of the pictures and derived DTM match the CIVA-P pictures. At the end of the mission, Rosetta may perform a close flyby of this site and will take pictures with a resolution allowing an unambiguous identification of Philae.

The SONC-FD landing site was chosen before consolidated data of $[10,11]$ were provided and better DTM available. According to $[10,11]$, it is clearly not the best site but it is close enough to all proposed area and sites. Moreover as the DTM used to realize the analyses is locally very smooth and the estimated attitude of Philae is correct up to a tens of degree, to move the position of Philae by a tens of meters would not change too much the prediction.

\subsection{Analysis of Philae position and orientation for wake-up date prediction}

Once the position and the attitude estimated, the situation and future of Philae was better understood with some good and bad news

The bad news was that the lander is almost lying on the side and its antenna is pointing toward the comet making communication difficult. More than $60 \%$ of the antenna half-lobe is obstructed by the ground. The area is extremely rocky and our local DTM too smooth to properly model the shadowing. Fig. 18 presents a view showing Philae (size magnified by 25) on its final landing site. The good news was that the lander was at more southern latitude than its targeted landing site and that the local illumination will improve in such a way that Philae may have

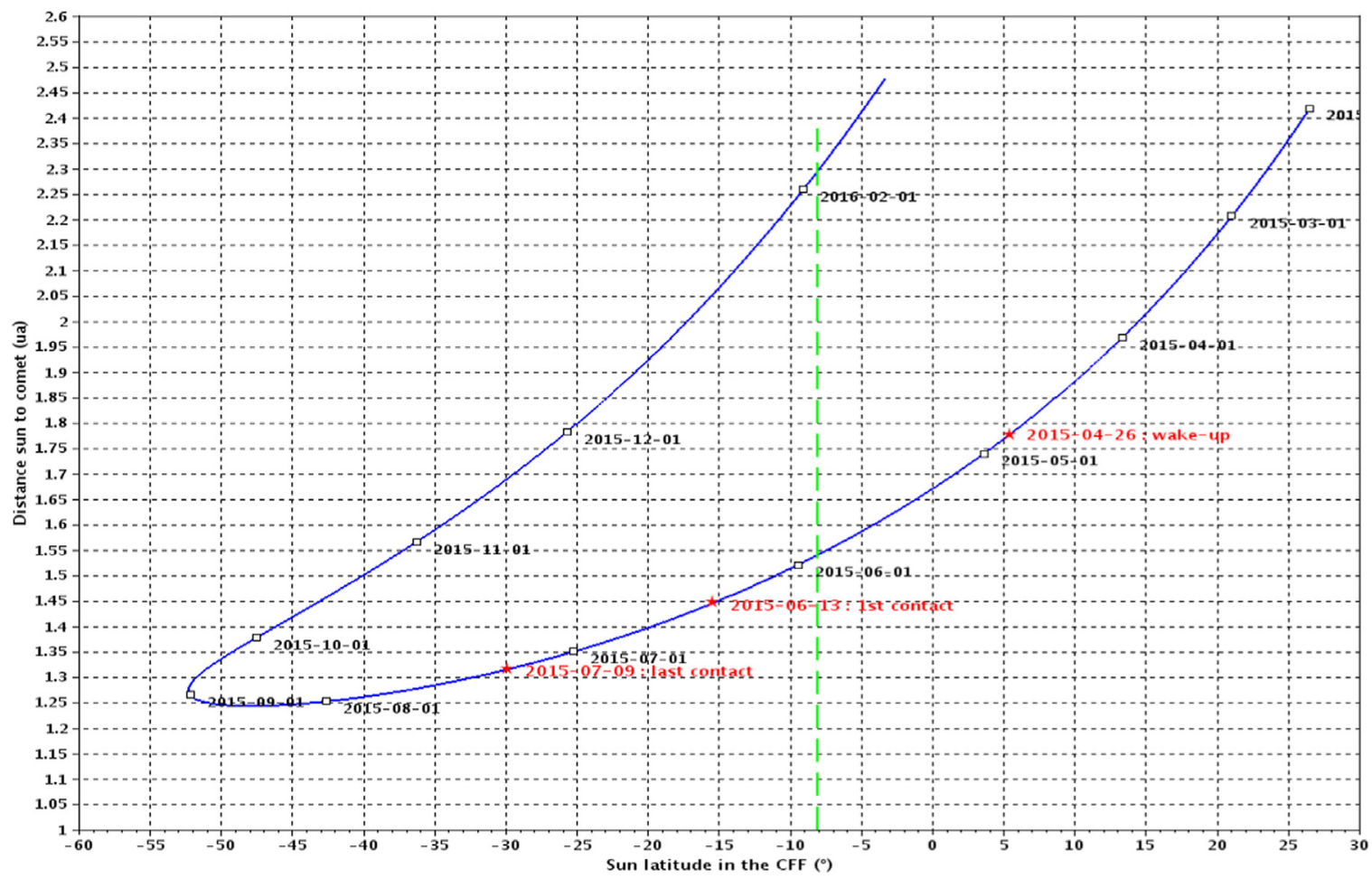

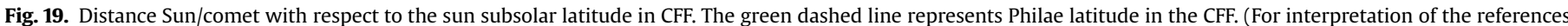
to color in this figure legend, the reader is referred to the web version of this article.) 
enough power to wake up. The improvement has two origins. First the distance Sun/comet was decreasing and the comet reached its perihelion (1.2 AU) the 13th of August 2015. As during FSS the distance was $3 \mathrm{AU}$, the photon flux reaching Philae will be multiplied by a factor 6 . Second improvement, the sun elevation with respect to the landing site grew up until June thanks to the seasonal effect.

Fig. 19 represents the distance Sun/comet with respect to the latitude of the subsolar points from February 2015 to February 2016. For a given date, the subsolar point represents the intersection between the comet surface and the direction from the comet center to the sun. The green dashed line represents the latitude of Philae. This plot indicates that at local noon the first of June, the sun reach its maximum elevation.

LCC analyzed the SONC-FD illumination prediction and concludes that Philae, if still working, could wake-up around April/ May 2015.

\section{Operations during wake-up phase}

A wake-up of Philae is possible only if the solar arrays produce at least $5.5 \mathrm{~W}$ and if the internal temperature exceeds $-45^{\circ} \mathrm{C}$. If this happens, Philae is able to detect a signal from Rosetta and to charge its battery. To communicate with Rosetta, Philae should at least have $19 \mathrm{~W}$ available and receive signal from Rosetta (i.e. the hibernation mode allows only to answer to Rosetta). Philae is not using its battery unless commanded.

Since February 2015, several communication campaigns were realized by Rosetta. Rosetta sent blinds commands to the lander. The purpose of the commands is to optimize the use of energy on board Philae. If Philae receives it, it can execute them even if it is unable to answer to Rosetta. if:

A communication between Rosetta and Philae is possible only

- The distance between the two spacecraft is not too large. The antennae were designed for communications within the $60^{\circ}$ half-lobe up to $80 \mathrm{~km}$ but may be possible up to $150 \mathrm{~km}$ most probably with $40 / 50^{\circ}$ half-lobe.

- Rosetta and Philae antennas should face each other.

- The lander should be illuminated since at least 15 min (time necessary to awake). Most of the time (for security reasons), Rosetta is moving along terminator orbits. It may fly over the lander either the morning, either the evening. As Philae required to be illuminated as long as possible, the morning path was chosen.

The 13th of June, Philae contacted briefly Rosetta. The contact was very short, only $85 \mathrm{~s}$, and unstable but 300 data packet were transmitted to Earth through Rosetta.

After an analysis of the transmitted HK, it appears that the lander started to awake the 26th of April (as explained earlier this date is quite approximate as it is computed thanks to the reboot increment).

Between the 13th of June and 9th of July, 8 others contacts occurred. The best one, almost $20 \mathrm{~min}$ long, was the last one (obtained with an orbit altitude of $150 \mathrm{~km}$ ). To improve the contact, ESA tried to decrease Rosetta's attitude but as the outgassing was increasing, orbits lower than $150 \mathrm{~km}$ altitude could not be reached. The outgassing creating problems with the star trackers, Rosetta had even to increase its altitude again.

After the wake-up, ESA dedicated Rosetta orbits to the communication with Philae. Rosetta was flying orbit through a zone of latitude defined by SONC-FD (taking into account the lander position and the antenna field of view). The orbit was crossing the

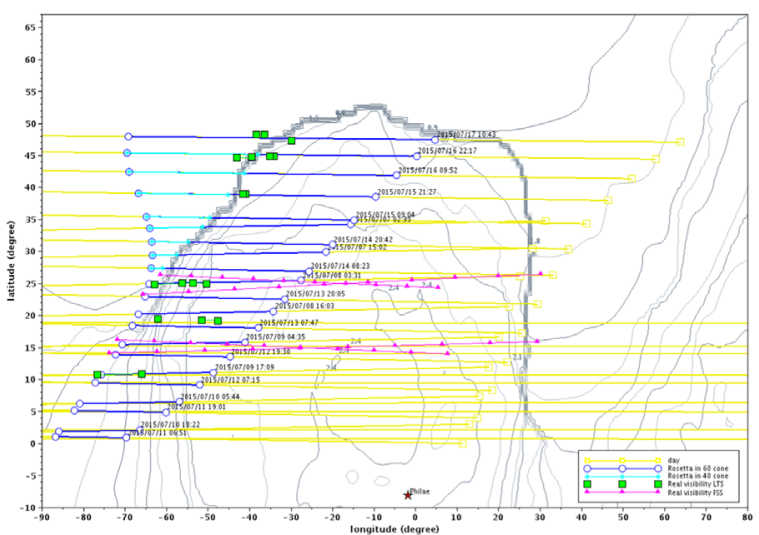

Fig. 20. Prediction of communication opportunities between Rosetta and Philae from 7th of July 2015 to 17 of July 2015. (For interpretation of the references to color in this figure legend, the reader is referred to the web version of this article.)

latitude band between $5^{\circ}$ and $55^{\circ}$. Rosetta was flying over Philae the morning (terminator orbit). Based on the Rosetta ephemeris and attitude provided by RMOC, SONC-FD was computing twice a week the communication opportunities. Fig. 20 is a typical output of the predictions. It is a topographic map (longitude, latitude, radius of the comet).

The signification of the colored lines and symbols are:

- The red star is the Philae position.

- The gray lines are the comet topography (i.e. radius).

- The yellow lines are the portion of the Rosetta ground tracks when Philae is illuminated.

- The dark blue lines are the portion of Rosetta orbits where communications are possible considering a $60^{\circ}$ half-lobe for the antenna of Philae and Rosetta.

- The light blue lines are the portion of Rosetta orbits where communications are possible but only with a $40^{\circ}$ half-lobe.

- The green squares represent the Rosetta ground tracks for real communication opportunities.

- The pink lines represent the Rosetta ground tracks for communications that occurred during the FSS.

We experienced less communications than expected. Rosetta was sometime flying almost on the same orbit than one where Philae communicated some days ago, but nothing happens.

This behavior was not explained but we suspect several origins:

- Some problems with the platform. One of the two Philae receivers does not work and one of the two transmitters works erratically. Some anomalies are also suspected at Lander mass memory level.

- The distance Rosetta/Philae is too large and received signals are very weak and close to the detection limits.

- Some rocks may obstruct portions of the Philae antenna field of view preventing any communications.

For the 15th July-15th October period, the outgassing level was too high for Rosetta to fly lower enough to ensure communications with the Lander. Nevertheless, there is good hope that in the coming months, contacts could be established again as Rosetta will come closer to the comet surface.

\section{Reconstruction of landing and bouncing trajectory}

In parallel, for scientific purpose, several teams worked on the reconstruction of the descent and bouncing trajectories. 
Table 8

Comet models used and effect on the landing site date and position.

\begin{tabular}{|c|c|c|c|c|c|}
\hline Model set & Shape Model & Gravity & Outgassing & $\begin{array}{l}\text { Time delay for the touchdown dates } \\
\text { (s) }\end{array}$ & $\begin{array}{l}\text { Distance to real touchdown site } \\
\text { (m) }\end{array}$ \\
\hline 1 & OSIRIS & RMOC spherical harmonic expansion & No & 10.1 & 7.3 \\
\hline 2 & RMOC & RMOC, spherical harmonic expansion & Yes & -2.37 & 12.1 \\
\hline 3 & OSIRIS & $\begin{array}{l}\text { Gravity field derived from the constant density } \\
\text { polyhedron }\end{array}$ & No & 5.1 & 11.1 \\
\hline
\end{tabular}

\subsection{Descent trajectory}

The descent trajectory may only be estimated from simulation. CONSERT radar was active during the descent and performed ranging measurements but its accuracy is not sufficient enough to constrain the descent trajectory. It only shows that Philae descent was very close to the targeted trajectory.

Based on ROLIS and CIVA images, CNES robotics team was able to derive the touchdown position [2,9] and attitude [9].

We then estimate the trajectory by using a classical propagation and taking into account:

- The epoch, position and velocity at the date of release (available from RMOC data).

- A shape model provided by OSIRIS. The shape model is a triangular faces polyhedron with $5 \mathrm{~m}$ resolution.

- A gravity field model. RMOC provided a classical spherical harmonics expansion (up to degree 3 ) derived from the navigation data. SONC-FD also used a gravity model derived from the shape model assuming a constant density [7].

- The comet ephemeris and rotation parameters provided by RMOC.

- A comet outgassing provided by RMOC. The outgassing was very small at the date of landing so we did not use it all the time.

We also have neglected the solar radiation pressure.

According to Table 8, largest distance of real landing site is $12 \mathrm{~m}$ and largest time delay is $10 \mathrm{~s}$. The main contributors to the propagation error are the inaccuracies of the point of release and the DTM errors around the landing site. For all models set, the impact velocity expressed in CFF is close to $1.01 \mathrm{~m} / \mathrm{s}$ (one notice that a $10 \mathrm{~m}$ error on the release point is around a $10 \mathrm{~s}$ delay in the landing date).

\subsection{Rebounds}

The results presented here are preliminary and need to be confirmed by more analyses.

The available data to rebuild the rebound trajectories are not numerous:

- The dates of touchdowns and final landing (cf. Table 1),

- an estimation of the position of the first touchdown and final landing position,

- two estimations of Philae position during the fly between touchdown 1 and 2. OSIRIS camera took some pictures of the landing zone. Philae and its shadows were observed on one picture and CNES robotics team was able to compute an estimate of the position [13].

- NAVCAM also observed Philae during second and third touchdown but we did not have the position estimation.

The comets models used for the analysis are:

- The comet ephemeris and rotation parameters provided by
RMOC,

- the shape model provided by OSIRIS (as in Section 4),

- the gravitational potential is computed by considering a constant density polyhedron. This method seems preferable than the RMOC spherical harmonics expansion because it represents better close vicinity comet gravity field (Spherical harmonics expansion is limited to degree 3 ) and is valid everywhere outside the comet surface (Spherical harmonic expansion may present divergence properties inside the smallest sphere enclosing the shape model).

- The outgassing and the solar radiation pressure are considered negligible.

The construction of a rebound trajectory was realized by optimizing a trajectory taken into account the available data. The optimization was based on the well-known Nelder-Mead algorithm.

The trajectory is optimized according to the following constraints:

- The flight duration of each rebound (plus or minus one minutes).

- At the date where the second OSIRIS picture was acquired, the lander should be at less $75 \mathrm{~m}$ from the position determined by CNES robotics team.

- The final landing site should be at less than $20 \mathrm{~m}$ from the determined ones.

We decided to not model the last rebound as its flying time was less than 6 min. In other words, we consider that the touchdown 3 is close enough to the real final landing site.

Among all trajectories that the optimizer could find, we extract 6 trajectories that are represented on Fig. 21. Touchdown 1 is identical for all cases and touchdown 2 is located on various part of the crater rim. The largest distance between two touchdown 2 site is $275 \mathrm{~m}$.

To characterize the rebound geometry we also computed the rebound angle (angle between $-\vec{V}_{i n}, \vec{V}_{\text {reb }}$ ) and the absorption coefficient $\frac{\left\|\vec{v}_{\text {reb }}\right\|}{\left\|\vec{v}_{\text {in }}\right\|}$.

Fig. 22 represents for each rebound the absorption coefficient with respect to the rebound angle.

Whatever the trajectory is, the two rebounds are different: for

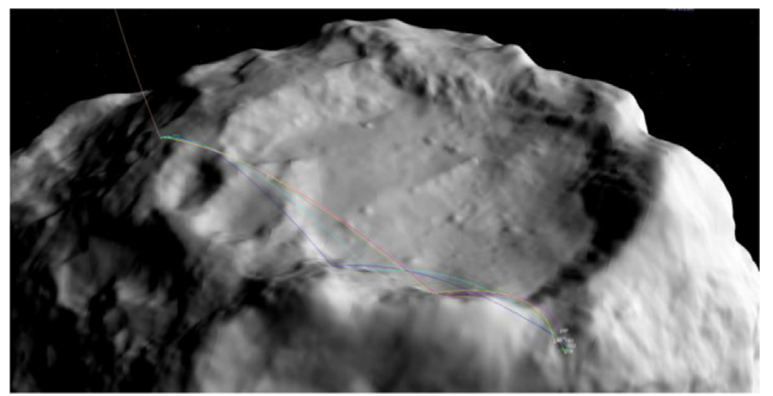

Fig. 21. 6 Rebound trajectories obtained through optimization. 


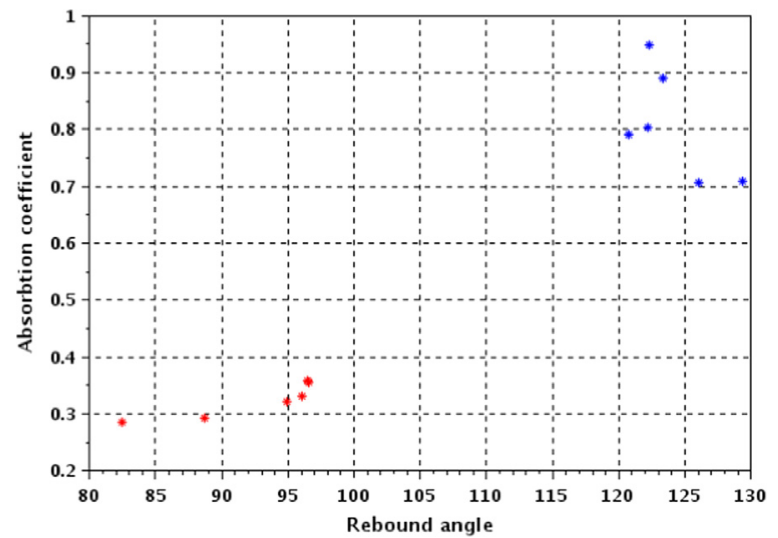

Fig. 22. Characterization of the rebound geometry for touchdown 1 and 2 .

first rebound the incoming velocity is almost along the normal to the touchdown ( $4^{\circ}$ angle) but the rebound velocity is nearly orthogonal to the incoming velocity. At moment of touchdown, the lander was rotating around its $Z$ axis and the three feet of the landing gear touch the ground one after the other [2]. As a consequence, it transforms a vertical velocity (relative to the local normal) to a tangential velocity. Two third of the incoming velocity (Vin $=1.01 \mathrm{~m} / \mathrm{s}$ ) is dissipated by the landing gear dumping mechanics and by the contact with the ground. This is clearly not an elastic collision.

Second touchdown exhibits a different behavior. The angle between the incoming and rebound velocities is more than $120^{\circ}$. The contact seems to be hard with low velocity absorption (absorption coefficient between 0.7 and 1 ). The collision is almost elastic.

Concerning the attitude determination, this task is still ongoing. The work to realize is equivalent to the one realized with ROMAP for the descent. As results are very preliminary, there are not presented here.

\section{Conclusion}

From August to November 2014, several loops of Flight Dynamics analyses have been carried out to determine the best final landing site for Philae lander. Landing Site "Agilkia" was finally chosen as the one presenting the less risk for the Lander delivery, and with good conditions for the Lander instruments. This site was reached with a very good accuracy on 12th November 2014 at $15 \mathrm{~h}$ $34 \mathrm{~min} 04 \mathrm{~s}$ but due to the failure of the anchoring system, Philae bounced off from comet surface and came to a rest about $1 \mathrm{~km}$ away from the targeted point, in a poorly illuminated area. After $7 \mathrm{~h}$ of descent and $57 \mathrm{~h}$ of on-comet operations, the primary battery was finally completely depleted and due to the lack of solar illumination on the solar arrays, the Lander went in hibernation mode.

Thanks to a collaborative effort involving various teams (instruments, operational teams...), the descent and bouncing trajectories as well as the orientation at the final landing site were reconstructed, leading to a better understanding of the current situation of Philae. These efforts were rewarded in June 2015 when Philae finally sent a signal to Rosetta, and new data were retrieved from the Lander TM. These new data confirmed the estimate of the position and orientation of our Lander.

Due to the very high level of outgassing of the comet near perihelion, Rosetta had to fly several hundred kilometers away from the comet, and contacts between Lander and Orbiter were then impossible. Today it is planned to reduce the distance starting mid-October, and to try to establish new contacts with Philae, in order to activate once again its instruments.

\section{References}

[1] E. Jurado, et al., LSSP, Landing Site and Descent Trajectory selection process, RLGS-TNO-LTAN-TECH-9318-CN, v2.0, July 2014.

[2] J. Biele, et al., RD-2000, Rosetta Lander FD data and models, RO-LAN-RD-2000, v4.5, December 2013.

[3] SONC-FD, Philae Flight Dynamics Mission Analysis, Science Operations and Navigation Center Flight Dynamics team, RLGS-TNO-OPER-9306-CN.

[4] Bobert A. Werner, The gravitational potential of a homogeneous polyhedron or donot cut corners, Celest. Mech. Dyn. Astron. 59 (1994) 253-278.

[5] F.S. Ulamec, et al., Rosetta landerations and navigation center, Acta Astronaut. 107 (2015) 77-86.

[7] J. Biele, et al., The landing(s) of Philae and inferences about comet surface mechanical properties, Science 349 (6247) (2015) aaa9816.

[8] P. Heinisch, et al., ROMAP, Attitude reconstruction of ROSETTA's lander PHILAE using two-point magnetic field observations by ROMAP and RPC-MAG, In preparation Acta Astronautica 125 (2016) 174-182.

[9] W. Kofman, et al., Properties of the 67P/Churyumov-Gerasimenko interior revealed by CONSERT radar, Science 349 (6247) (2015) aab0639.

[10] A. Herique, et al., Philae localization from CONSERT/ROSETTA measurement, Planet. Space Sci. 117 (2015) 475-448.

[11] P. Lamy, et al., Identification and characterization of the landing site of Philae from OSIRIS-NAC Images, in: Proceedings of the EPSC Abstracts, vol. 10, EPSC2015-783, 2015

[13] E. Remetean, et al., Philae locating and science support by robotic vision techniques, In preparation Acta Astronautica 125 (2016) 161-173.

[16] B. Davenport, A vector approach to the algebra of rotations with applications, NASA technical note, NASA-TN D-4696, 1968.

[17] Reinhard Roll, ROSETTA Lander Philae tor approach to the algebra of roh-down 1 reconstruction, Lars Witte, In preparation Acta Astronautica 125 (2016) 149-160. 